\begin{tabular}{|c|l|}
\hline Title & THE MOTION OF ELA STIC PLA NA R CLOSED CURV ES UNDER THE AREA-PRESERV ING CONDITION \\
\hline Author(s) & Okabe, Shinya \\
\hline Citation & Hokkaido University Preprint Series in Mathematics, 812, 1-31 \\
\hline Issue Date & 2006 \\
\hline DOI & 10.14943/83962 \\
\hline Doc URL & http://hdl.handle.net/2115/69620 \\
\hline Type & bulletin (article) \\
\hline File Information & pre812.pdf \\
\hline
\end{tabular}

Instructions for use 


\title{
THE MOTION OF ELASTIC PLANAR CLOSED CURVES UNDER THE AREA-PRESERVING CONDITION
}

\author{
SHINYA OKABE
}

\begin{abstract}
We consider the motion of an elastic closed curve with constant enclosed area. This motion is governed by a system involving fourth order parabolic equations. We shall prove that this system has a unique classical solution for all time and the solution converges uniformly to a stationary solution together with its derivatives of any order.
\end{abstract}

\section{INTRODUCTION}

The variational problem for the total squared curvature functional defined on curves has been studied since the middle of the 18th century. Euler considered this problem for curves of a fixed length, and its solution is called an elastica. Since then, there have been many studies in this field, for example, on a closed elastica ([6], [7]), a buckled elastic ring ([2], [15]), a $L^{2}$-gradient flow for the total squared curvature ([13]), a curve straightening flow ([8], [9], [10], [16], [17]), the motion of an elastic curve $([5])$, as well as an area-preserving elastica in spectral geometry ([11], [18], [19]). In this paper, we consider the motion of an elastic curve under two constraints, which can also be viewed as the gradient flow towards area-preserving elasticae.

Let us consider a simple closed curve made of springy wire in the plane. The inner domain enclosed by the wire and its exterior are filled with two kinds of incompressible viscous fluids $F_{i}$ and $F_{o}$, respectively. The wire is expected to change its shape so as to decrease its bending energy as efficiently as possible. On the other hand, by the assumption of incompressibility, the area of the inner domain is preserved. We are interested in the following question: "Can the wire change its shape so as to decrease its bending energy and preserve its enclosed area? Moreover, if it can, what shape will the wire become eventually?" The purpose of this paper is to analyze the dynamics of such a wire.

We regard the wire as a closed plane curve $\gamma: S_{L}^{1} \rightarrow \mathbb{R}^{2}$, where $S_{L}^{1}:=\mathbb{R} / L \mathbb{Z}$ and $L$ denotes its length. The motion of $\gamma$ is governed by the gradient flow for the total squared curvature

$$
E(\gamma)=\oint \kappa^{2}(s) d s
$$

subject to the following two constraints:

(C1) $\quad \gamma$ is inextensible;

(C2) the area enclosed by $\gamma$ is preserved.

Date: 2006 .

1991 Mathematics Subject Classification. 74H40 (35K55, 74G65, 74B20).

Key words and phrases. elastic energy, gradient flow with two constraints, isoperimetric inequality. 
Here, $\kappa$ and $s$ denote the curvature of $\gamma$ and the arc-length parameter, respectively. We call $E$ the elastic energy of $\gamma$. By the area enclosed by $\gamma$, we mean the signed area defined by $(3.3)$.

We shall derive in Section 3 the following system of equations that governs the gradient flow for the elastic energy $E$ under two constraints (C1) and (C2):

$$
\left\{\begin{array}{l}
\frac{\partial \gamma}{\partial t}=-\frac{\partial^{4} \gamma}{\partial x^{4}}+\frac{\partial}{\partial x}\left\{\left(v-2\left|\frac{\partial^{2} \gamma}{\partial x^{2}}\right|^{2}\right) \frac{\partial \gamma}{\partial x}\right\}+\lambda \boldsymbol{n}, \\
-\frac{\partial^{2} v}{\partial x^{2}}+\left|\frac{\partial^{2} \gamma}{\partial x^{2}}\right|^{2} v=2\left|\frac{\partial^{2} \gamma}{\partial x^{2}}\right|^{4}-\left|\frac{\partial^{3} \gamma}{\partial x^{3}}\right|^{2}-\lambda \boldsymbol{n} \cdot \frac{\partial^{2} \gamma}{\partial x^{2}} \\
\int_{0}^{L}\left\{\left(\boldsymbol{n} \cdot \frac{\partial^{2} \gamma}{\partial x^{2}}\right) v-\left(\boldsymbol{n} \cdot \frac{\partial^{2} \gamma}{\partial x^{2}}\right)^{3}+\lambda\right\} d x=0,
\end{array}\right.
$$

where $v(x, t)$ and $\lambda(t)$ are unknown scalar functions. Here $t$ denotes the time variable and $\boldsymbol{n}(x, t)$ denotes the unit normal vector of $\gamma(x, t)$ given by the formula $\boldsymbol{n}=R \gamma^{\prime}$ with $R=\left(\begin{array}{cc}0 & -1 \\ 1 & 0\end{array}\right)$, where $\gamma^{\prime}$ is the unit tangent vector of $\gamma$.

We find $(\gamma(x, t), v(x, t), \lambda(t))$ which satisfies $(\mathrm{GT})$ with an initial data. However we impose an initial condition only on $\gamma: \gamma(x, 0)=\gamma_{0}(x)$. When $\gamma_{0}(x)$ is not a circle, we do not prescribe $v(x, 0)=v_{0}(x)$ and $\lambda(0)=\lambda_{0}$, because $\gamma(x, t)$ determines $v(x, t)$ and $\lambda(t)$ uniquely (see Lemma 4.1). On the other hand, when $\gamma_{0}(x)$ is a circle with radius $r$, for any continuous function $\lambda_{0}(t),(\gamma(x, t), v(x, t), \lambda(t))=\left(\gamma_{0}(x), 1 / r^{2}-\lambda_{0}(t) r, \lambda_{0}(t)\right)$ satisfies (GT). Therefore we do not prescribe initial data for $v(x, t)$ and $\lambda(t)$ either in this case.

The main result of the paper is stated as follows:

Theorem 1.1. Let $\gamma_{0}(x)$ be a smooth closed curve with length $L$, enclosed area $\mathcal{A}_{0}$ and rotation number 1 . In addition, assume that $\gamma_{0}(x)$ is not a circle. Suppose that $\gamma_{0}(x)$ satisfies $\left|\left(\partial \gamma_{0} / \partial x\right)(x)\right| \equiv 1$. Then (GT) has a unique classical solution $(\gamma(x, t), v(x, t), \lambda(t))$ for all $t>0$, and the solution $\gamma(x, t)$ satisfies $|(\partial \gamma / \partial x)(x, t)| \equiv 1$. Moreover, when $t \rightarrow \infty$, $(\gamma(x, t), v(x, t), \lambda(t))$ converges to a solution $(\hat{\gamma}(x), \hat{v}(x), \hat{\lambda})$ of the equation

$$
\left\{\begin{array}{l}
-\frac{\partial^{4} \gamma}{\partial x^{4}}+\frac{\partial}{\partial x}\left\{\left(v-2\left|\frac{\partial^{2} \gamma}{\partial x^{2}}\right|^{2}\right) \frac{\partial \gamma}{\partial x}\right\}+\lambda \boldsymbol{n}=0, \\
-\frac{\partial^{2} v}{\partial x^{2}}+\left|\frac{\partial^{2} \gamma}{\partial x^{2}}\right|^{2} v=2\left|\frac{\partial^{2} \gamma}{\partial x^{2}}\right|^{4}-\left|\frac{\partial^{3} \gamma}{\partial x^{3}}\right|^{2}-\lambda \boldsymbol{n} \cdot \frac{\partial^{2} \gamma}{\partial x^{2}} \\
\int_{0}^{L}\left\{\left(\boldsymbol{n} \cdot \frac{\partial^{2} \gamma}{\partial x^{2}}\right) v-\left(\boldsymbol{n} \cdot \frac{\partial^{2} \gamma}{\partial x^{2}}\right)^{3}+\lambda\right\} d x=0,
\end{array}\right.
$$

in the $C^{\infty}$ topology.

When $\gamma_{0}(x)$ is a circle, we have the following.

Proposition 1.1. Let $\gamma_{0}(x)=\vec{a}+r\left(\cos \frac{x}{r}, \sin \frac{x}{r}\right)$, where $\vec{a}$ is a constant vector and $r$ is a positive constant. For each continuous function $\lambda_{0}(t)$, (GT) has a unique solution $(\gamma(x, t), v(x, t), \lambda(t))=\left(\gamma_{0}(x), 1 / r^{2}-\lambda_{0}(t) r, \lambda_{0}(t)\right)$. Moreover, this $(\gamma, v, \lambda)$ also satisfies $(\mathrm{AE})$. 
We mention here results which are closely related to this paper. A. Polden ([13]) considered the $L^{2}$-gradient flow for the elastic energy without a constraint. The regularity estimates for a solution of (GT), which are used in Section 5, are inspired by [13]. Y. Wen ([16], [17]) considered the flow of closed curves with fixed length along the negative $L^{2}$-gradient flow of the elastic energy. On the other hand, N. Koiso ([5]) considered the motion of an elastic closed curve in $\mathbb{R}^{3}$, i.e., the negative gradient flow of the elastic energy subject to the single constraint $(\mathrm{C} 1)$, which is the inextensibility condition. The derivation of the system of equations (GT) is similar to that of [5]. The gradient flow in [5] is governed by a system consisting of the first two equations in (GT) with $\lambda=0$. He proved that this system has a unique classical solution for all $t>0$, and the solution converges to an elastica as $t \rightarrow \infty$ in the $C^{\infty}$ topology.

A difference between this paper and [5] is the area-preserving condition $(\mathrm{C} 2)$, which brings an additional unknown function $\lambda(t)$. By $(\mathrm{C} 2)$, we immediately see that a circle is singular to our problem, i.e., if the initial data $\gamma_{0}(x)$ is a circle, in order to keep the length and the area constant, $\gamma(x, t)$ must be a circle of the same radius for all $t>0$ because of the isoperimetric inequality. Therefore we stated our results separately. When $\gamma_{0}(x)$ is not a circle, imposing the second constraint (C2) raises two new mathematical questions. First, it is natural to ask whether the system (GT) has any solution at least on a short time interval or not. One of the main contributions of this paper is to give an affirmative answer to this question. Indeed, we can construct a mild solution in the space $C\left([0, T] ;\left(W^{4, p}\left(S_{L}^{1}\right)\right)^{2} \times W^{2,2}\left(S_{L}^{1}\right) \times \mathbb{R}\right)$ with $p \geq 3$ and show that it is a classical solution. (For the definition of $W^{m, q}$, see Section 2.) In doing so, we decompose $v(x, t)$ as $v(x, t)=u(x, t)+\lambda(t) w(x, t)$ and obtain the expression

$$
\lambda(t)=\frac{\int_{0}^{L}\left\{-\kappa(x, t) u(x, t)+\kappa^{3}(x, t)\right\} d x}{L+\int_{0}^{L} \kappa(x, t) w(x, t) d x},
$$

where $u(x, t)$ and $w(x, t)$ are determined by $\gamma(x, t)$ uniquely. The denominator is positive whenever $\gamma(x, t)$ is not a circle (see Lemma 4.1.) Hence the existence of a unique mild solution is guaranteed for $T>0$ sufficiently small by the continuity of $\kappa(x, t)$ and $w(x, t)$ in the case where $\gamma_{0}(x)$ is not a circle. A second question is whether the denominator in (1.2) vanishes in finite time (or converges to 0 as $t \rightarrow \infty$ ) or not. We shall prove that the denominator is bounded away from 0 (see Lemma 5.4), hence the solution exists for all $t>0$ and remains bounded. We note that the isoperimetric inequality plays a crucial role in the proof of Lemma 5.4. The convergence of the solution as $t \rightarrow \infty$ can be verified in a way analogous to that in [5].

As mentioned above, there are many studies on the variational problem for the energy (1.1). For closed curves, Langer and Singer [6] have completely classified the closed elasticae and determined the knot types of elasticae.

There are a few studies on an area-preserving elastica, which corresponds to the stationary problem for our problem. For example, it has been studied by K. Watanabe ([18], [19]). In [18], he has proved the existence of a minimizer $\gamma$, which is a smooth simple closed curve and has an even number of axes of symmetry, under the assumption $4 L^{2} / 25 \pi \leq M \leq L^{2} / 4 \pi$. Here, $L$ and $M$ denote the perimeter of $\gamma$ and the area enclosed by $\gamma$, respectively. In [19], by assuming $0<L^{2} / 4 \pi-M<\delta_{0}$, where $\delta_{0}$ is sufficiently small, he proved further that the minimizer $\gamma$ is an oval with four vertices. Recently, Matsumoto, Murai, and Yotsutani [11] derived explicit representations of the curvature $\kappa$ 
of $\gamma$ for each $n \geq 2$, where $\gamma$ is the solution of this variational problem and $n$ is a number of axes of symmetry of $\gamma$. They assume the rotation number of $\gamma$ to be one. Since the Euler-Lagrange equation in terms of $\kappa$ becomes

$$
\frac{d^{2} \kappa}{d s^{2}}+\frac{1}{2} \kappa^{3}-\mu_{1} \kappa-\mu_{2}=0
$$

where $\mu_{1}$ and $\mu_{2}$ are the Lagrange multipliers, $\kappa$ can be represented by Jacobian elliptic functions. We shall prove in Section 3 that (AE), which is the stationary problem for (GT), is equivalent to (1.3).

It may be interesting to look on our problem as a lower dimensional analogue to Canham's variational problem for the total squared mean curvature (the Willmore functional) of a (two dimensional) closed surface. Canham [1] proposed this variational problem as a model of the shape of a red blood cell. See also [4] for a dynamical aspect of this variational problem.

This paper is organized as follows: In Section 2, we state several lemmas often used in this paper without proof, which have been proved in [5]. In Section 3, we formulate our problem and derive the system of equations (GT). The proof of Theorem 1.1 breaks into the following three parts: In Sections 4 and 5, we prove the short time existence and long time existence, respectively; finally, we prove the convergence of the solution of (GT) in Section 6. Moreover, we prove Proposition 1.1 also in Section 6.

Acknowledgments. The author would like to thank Professor I. Takagi of Tohoku University for many useful suggestions.

\section{Preliminaries}

First, we define several notations as follows:

$$
\dot{\gamma}=\frac{\partial \gamma}{\partial t}, \quad \gamma^{\prime}=\frac{\partial \gamma}{\partial x}, \quad \gamma^{(n)}=\frac{\partial^{n} \gamma}{\partial x^{n}} .
$$

We sometimes use these notations for short. Let us define $W^{m, q}\left(S_{L}^{1}\right)$ to be the usual Sobolev space on $S_{L}^{1}$, i.e. the Banach space of functions whose distributional derivatives up to order $m$ belong to $L^{q}\left(S_{L}^{1}\right)$.

We shall state several lemmas often used in this paper without proof. These lemmas have been proved in [5].

Lemma 2.1 (N. Koiso [5]). Let $a(x)$ and $f(x)$ be continuous functions on $S_{L}^{1}$ and assume that $a(x) \geq 0$ and $\|a\|_{L^{1}}>0$. Then, $-v^{\prime \prime}+a v=f$ has a unique solution. Moreover, the solution $v$ satisfies

$$
\begin{aligned}
& \max _{x \in S_{L}^{1}}|v| \leq\left(2 L+\|a\|_{L^{1}}^{-1}\right)\|f\|_{L^{1}}, \\
& \max _{x \in S_{L}^{1}}\left|\frac{d v}{d x}\right| \leq 2\left(1+L\|a\|_{L^{1}}\right)\|f\|_{L^{1}} .
\end{aligned}
$$

Lemma 2.2 (N. Koiso [5]). Let $v$ be the solution of $-v^{\prime \prime}+a v=f$ on $S_{L}^{1}$. Suppose that $a \geq 0$ and there exists a positive constant $\delta$ such that $\|a\|_{L^{1}} \geq \delta$. Then the solution $v$ 
satisfies

$$
\begin{aligned}
& \|v\|_{C^{n+2}} \leq C\left(1+\|a\|_{C^{n}}^{N}\right)\|f\|_{C^{n}}, \\
& \|v\|_{W^{n+2,2}} \leq C\left(1+\|a\|_{W^{n, 2}}^{N}\right)\|f\|_{W^{n, 2}},
\end{aligned}
$$

where $N$ and $C$ depend only on $n, L$ and $\delta$.

Lemma 2.3 (N. Koiso [5]). Let $v$ be an $\mathbb{R}^{N}$ valued function on $S_{L}^{1}$. Then we have

$$
\sup _{x \in S_{L}^{1}}|v|^{2} \leq 2\|v\|_{L^{2}}\left(\|v\|_{L^{2}}+\left\|\frac{\partial v}{\partial x}\right\|_{L^{2}}\right) \text {. }
$$

Moreover, when $\int_{0}^{L} v d x=0$, it holds that

$$
\sup _{x \in S_{L}^{1}}|v|^{2} \leq 2\|v\|_{L^{2}}\left\|\frac{\partial v}{\partial x}\right\|_{L^{2}} .
$$

For integers $0 \leq i \leq j \leq k$, it holds that

$$
\left\|\frac{\partial^{j} v}{\partial x^{j}}\right\|_{L^{2}} \leq\left\|\frac{\partial^{i} v}{\partial x^{i}}\right\|_{L^{2}}^{(k-j) /(k-i)}\left\|\frac{\partial^{k} v}{\partial x^{k}}\right\|_{L^{2}}^{(j-i) /(k-i)} .
$$

For integers $0 \leq i \leq j<k$ and $j>0$, it holds that

$$
\sup _{x \in S_{L}^{1}}\left|\frac{\partial^{j} v}{\partial x^{j}}\right| \leq\left\|\frac{\partial^{i} v}{\partial x^{i}}\right\|_{L^{2}}^{(2(k-j)-1) / 2(k-i)}\left\|\frac{\partial^{k} v}{\partial x^{k}}\right\|_{L^{2}}^{(2(j-i)+1) / 2(k-i)} .
$$

Lemmas 2.1 and 2.2 are simple modification of Lemmas 4.1 and 4.2 in [5]. We change $S^{1}=\mathbb{R} / \mathbb{Z}$ into $S_{L}^{1}=\mathbb{R} / L \mathbb{Z}$. We omit the proof.

\section{Formulation}

Let $\gamma_{0}(x)$ be a smooth closed plane curve parametrized by the arc-length parameter $x \in S_{L}^{1}$, so that $\left|\gamma_{0}{ }^{\prime}(x)\right| \equiv 1$. Let $L$ and $\mathcal{A}_{0}$ be the length of $\gamma_{0}$ and the signed area enclosed by $\gamma_{0}$, respectively. (For the precise definition of $\mathcal{A}_{0}$, see (3.3).) By the isoperimetric inequality, $L$ and $\mathcal{A}_{0}$ satisfy $L^{2} \geq 4 \pi\left|\mathcal{A}_{0}\right|$. We consider the smooth deformation $\gamma(\cdot, t)$ of the curve $\gamma_{0}$ depending on the time variable $t \geq 0$. Let $\gamma(x, t)$ denote the position at time $t$ of the point which was initially located at $\gamma_{0}(x): \gamma(x, 0)=\gamma_{0}(x)$. Suppose that $\gamma(x, t)$ satisfies two constraints (C1) and (C2). Since $x$ is the arc-length parameter of $\gamma_{0}$, the inextensibility condition $(\mathrm{C} 1)$ is expressed as

$$
\left|\frac{\partial \gamma}{\partial x}(x, t)\right| \equiv 1
$$

This in turn yields that $x$ is the arc-length parameter of $\gamma(\cdot, t)$ for any $t \geq 0$, and hence the length of $\gamma$ is given by $L$. Then the elastic energy $E$ is given by

$$
E(\gamma(x, t))=\int_{0}^{L}\left|\frac{\partial^{2} \gamma}{\partial x^{2}}(x, t)\right|^{2} d x .
$$

Moreover the signed area enclosed by $\gamma(x, t)$ is expressed as

$$
\mathcal{A}(\gamma(x, t))=-\frac{1}{2} \int_{0}^{L} \boldsymbol{n}(x, t) \cdot \gamma(x, t) d x
$$


Here $\boldsymbol{n}(x, t)$ denotes the unit normal vector of $\gamma(x, t)$, by which we mean that $\boldsymbol{n}=R \gamma^{\prime}$ with $R=\left(\begin{array}{cc}0 & -1 \\ 1 & 0\end{array}\right)$. (If $\gamma(x, t)$ is a simple closed curve and it is parametrized counterclockwise, then (3.3) gives the positive area.) Thus the area-preserving condition (C2) is given by

$$
-\frac{1}{2} \int_{0}^{L} \boldsymbol{n}(x, t) \cdot \gamma(x, t) d x \equiv \mathcal{A}_{0}
$$

We consider the gradient flow for the elastic energy (3.2) under the constraints (3.1) and (3.4). First we derive "the direction" that maximizes the decrease of the elastic energy. Since

$$
\frac{d}{d t} E(\gamma(x, t))=2 \int_{0}^{L} \frac{\partial^{2} \gamma}{\partial x^{2}}(x, t) \cdot \frac{\partial^{2}}{\partial x^{2}} \frac{\partial \gamma}{\partial t}(x, t) d x=2 \int_{0}^{L} \frac{\partial^{4} \gamma}{\partial x^{4}}(x, t) \cdot \frac{\partial \gamma}{\partial t}(x, t) d x
$$

"the direction" $\dot{\gamma}$ is given by $\dot{\gamma}=-\gamma^{(4)}$. However this direction does not fulfill two constraints (3.1) and (3.4). Therefore we wish to modify "the direction" by adding some terms. The condition (3.1) implies $\gamma^{\prime} \cdot \dot{\gamma}^{\prime}=0$. Hence, $\dot{\gamma}$ needs to be in the space

$$
V(t)=\left\{\begin{array}{l|l}
\eta \in W^{1,2}\left(S_{L}^{1} ; \mathbb{R}^{2}\right) & \frac{\partial \eta}{\partial x}(x) \cdot \frac{\partial \gamma}{\partial x}(x, t)=0
\end{array}\right\} .
$$

On the other hand, since $\int_{0}^{L} \dot{\boldsymbol{n}} \cdot \gamma d x=\int_{0}^{L} R \dot{\gamma}^{\prime} \cdot \gamma d x=-\int_{0}^{L} \dot{\gamma}^{\prime} \cdot R \gamma d x=\int_{0}^{L} \dot{\gamma} \cdot R \gamma^{\prime} d x=$ $\int_{0}^{L} \dot{\gamma} \cdot \boldsymbol{n} d x$, the condition (3.4) holds if and only if $\langle\partial \gamma / \partial t, \boldsymbol{n}\rangle_{L^{2}}=0$, where $\langle\mathbf{u}, \mathbf{v}\rangle_{L^{2}}=$ $\int_{0}^{L} \mathbf{u}(x) \cdot \mathbf{v}(x) d x$ for $\mathbf{u}, \mathbf{v} \in L^{2}\left(S_{L}^{1} ; \mathbb{R}^{2}\right)$. Hence we define the vector space $W$ by

$$
W(t)=\left\{\eta \in L^{2}\left(S_{L}^{1} ; \mathbb{R}^{2}\right) \mid\langle\eta, \boldsymbol{n}(\cdot, t)\rangle_{L^{2}}=0\right\} .
$$

If $\gamma(x, t)$ satisfies the constraints (3.1) and (3.4), then $\dot{\gamma} \in V(t) \cap W(t)$ for all $t \geq 0$. The orthogonal complements of $V$ and $W$ with respect to the $L^{2}$ inner product are given by

$$
\begin{aligned}
V^{\perp} & =\left\{\eta \in W^{1,2}\left(S_{L}^{1} ; \mathbb{R}^{2}\right) \mid \eta=\frac{\partial}{\partial x}\left(\xi(x, t) \frac{\partial \gamma}{\partial x}(x, t)\right) \text { for a certain scalar function } \xi\right\}, \\
W^{\perp} & =\left\{\eta \in L^{2}\left(S_{L}^{1} ; \mathbb{R}^{2}\right) \mid \eta=\lambda(t) \boldsymbol{n}(x, t) \text { for a certain scalar function } \lambda\right\},
\end{aligned}
$$

respectively. Since $(V \cap W)^{\perp}=V^{\perp}+W^{\perp}$, there exist some scalar functions $\xi(x, t)$ and $\lambda(t)$ such that $-\gamma^{(4)}+\left(\xi \gamma^{\prime}\right)^{\prime}+\lambda \boldsymbol{n} \in V \cap W$. Therefore we have

$$
\left\{\begin{array}{l}
\frac{\partial \gamma}{\partial t}=-\frac{\partial^{4} \gamma}{\partial x^{4}}+\frac{\partial}{\partial x}\left(\xi \frac{\partial \gamma}{\partial x}\right)+\lambda \boldsymbol{n} \\
\left\{-\frac{\partial^{5} \gamma}{\partial x^{5}}+\frac{\partial^{2}}{\partial x^{2}}\left(\xi \frac{\partial \gamma}{\partial x}\right)+\lambda \frac{\partial \boldsymbol{n}}{\partial x}\right\} \cdot \frac{\partial \gamma}{\partial x}=0 \\
\int_{0}^{L}\left\{-\frac{\partial^{4} \gamma}{\partial x^{4}}+\frac{\partial}{\partial x}\left(\xi \frac{\partial \gamma}{\partial x}\right)+\lambda \boldsymbol{n}\right\} \cdot \boldsymbol{n} d x=0 \\
\left|\frac{\partial \gamma}{\partial x}\right| \equiv 1
\end{array}\right.
$$

where the second and third equations mean $\dot{\gamma} \in V$ and $\dot{\gamma} \in W$, respectively. We claim that these equations are equivalent to the system (GT). First we consider the second equation. We use the relations obtained by differentiating (3.1) up to four times with 
respect to $x$ and the Frenet-Serret formula: $\gamma^{\prime \prime}=\kappa \boldsymbol{n}$ and $\boldsymbol{n}^{\prime}=-\kappa \gamma^{\prime}$. Then the second equation becomes as follows:

$$
2 \frac{\partial^{2}}{\partial x^{2}}\left(\left|\frac{\partial^{2} \gamma}{\partial x^{2}}\right|^{2}\right)-\left|\frac{\partial^{3} \gamma}{\partial x^{3}}\right|^{2}+\frac{\partial^{2} \xi}{\partial x^{2}}-\xi\left|\frac{\partial^{2} \gamma}{\partial x^{2}}\right|^{2}-\lambda \boldsymbol{n} \cdot \frac{\partial^{2} \gamma}{\partial x^{2}}=0
$$

Setting $v=\xi+2\left|\gamma^{\prime \prime}\right|^{2}$, we have the second equation of (GT).

Next we turn to the third equation. By the Frenet-Serret formula, we can transform the left-hand side of the third equation as follows:

$$
\begin{aligned}
\int_{0}^{L}\left\{-\frac{\partial^{4} \gamma}{\partial x^{4}}+\frac{\partial}{\partial x}\left(\xi \frac{\partial \gamma}{\partial x}\right)+\lambda \boldsymbol{n}\right\} \cdot \boldsymbol{n} d x & =\int_{0}^{L}\left\{\kappa v-\kappa^{3}+\lambda\right\} d x \\
& =\int_{0}^{L}\left\{\left(\boldsymbol{n} \cdot \frac{\partial^{2} \gamma}{\partial x^{2}}\right) v-\left(\boldsymbol{n} \cdot \frac{\partial^{2} \gamma}{\partial x^{2}}\right)^{3}+\lambda\right\} d x
\end{aligned}
$$

Therefore we obtain (GT) as desired.

Remark 3.1. Since we derive the second equation of (GT) as a necessary condition for the inextensibility condition (3.1), we need to check that a solution of (GT) satisfies the condition (3.1). On the other hand, a solution of (GT) satisfies the area-preserving condition (3.4) if and only if the third equation of (GT) holds.

We now prove that (AE) is equivalent to (1.3). By the first equation of (AE), we have

$$
0=\left\{-\gamma^{(4)}+\left(\left(v-2\left|\gamma^{\prime \prime}\right|^{2}\right) \gamma^{\prime}\right)^{\prime}+\lambda \boldsymbol{n}\right\} \cdot \gamma^{\prime}=\frac{3}{2}\left(\left|\gamma^{\prime \prime}\right|^{2}\right)^{\prime}+\left(v-2\left|\gamma^{\prime \prime}\right|^{2}\right)^{\prime} .
$$

Then the first equation of (AE) becomes as follows:

$$
-\gamma^{(4)}+\left(\left(C-\frac{3}{2}\left|\gamma^{\prime \prime}\right|^{2}\right) \gamma^{\prime}\right)^{\prime}+\lambda \boldsymbol{n}=0,
$$

where $C$ is a certain constant. Therefore we obtain

$$
0=\left\{-\gamma^{(4)}+\left(\left(C-\frac{3}{2}\left|\gamma^{\prime \prime}\right|^{2}\right) \gamma^{\prime}\right)^{\prime}+\lambda \boldsymbol{n}\right\} \cdot \boldsymbol{n}=-\kappa^{\prime \prime}-\frac{1}{2} \kappa^{3}+C \kappa+\lambda .
$$

This equation is nothing but (1.3).

\section{Short time EXISTEnCE}

In this section, we shall prove that (GT) has a unique classical solution on some time interval $[0, T)$. As a first step, we shall construct a mild solution of (GT). For this purpose, we use a successive approximation method in $C\left([0, T] ;\left(W^{4, p}\left(S_{L}^{1}\right)\right)^{2} \times W^{2,2}\left(S_{L}^{1}\right) \times \mathbb{R}\right)$ with $p \geq 3$ and the contraction mapping principle. Before describing the successive approximation scheme, we show that $v(x, t)$ and $\lambda(t)$ are determined by the second and third equations of (GT) for each time $t \geq 0$. 
Lemma 4.1. Suppose that $\gamma(x, t)$ satisfies $\left|\gamma^{\prime}(x, t)\right| \equiv 1$. Then

$$
\left\{\begin{array}{l}
-\frac{\partial^{2} v}{\partial x^{2}}+\left|\frac{\partial^{2} \gamma}{\partial x^{2}}\right|^{2} v=2\left|\frac{\partial^{2} \gamma}{\partial x^{2}}\right|^{4}-\left|\frac{\partial^{3} \gamma}{\partial x^{3}}\right|^{2}-\lambda \boldsymbol{n} \cdot \frac{\partial^{2} \gamma}{\partial x^{2}} \\
\int_{0}^{L}\left\{\left(\boldsymbol{n} \cdot \frac{\partial^{2} \gamma}{\partial x^{2}}\right) v-\left(\boldsymbol{n} \cdot \frac{\partial^{2} \gamma}{\partial x^{2}}\right)^{3}+\lambda\right\} d x=0
\end{array}\right.
$$

has a solution $(v(x, t), \lambda(t))$ for each time $t \geq 0$. If $\gamma(x, t)$ is not a circle, (4.1) has a unique solution $(v(x, t), \lambda(t))$. On the other hand, if $\gamma(x, t)$ is a circle with radius $r$, then the set of the solutions is expressed as

$$
\left\{(v, \lambda) \mid v=\frac{1}{r^{2}}-\lambda r, \lambda \in \mathbb{R}\right\}
$$

Proof. Let $\kappa$ be the curvature of $\gamma$. Then (4.1) becomes as follows:

$$
\left\{\begin{array}{l}
-v^{\prime \prime}+\kappa^{2} v=\kappa^{4}-\left(\kappa^{\prime}\right)^{2}-\lambda \kappa \\
L \lambda=-\int_{0}^{L}\left(\kappa v-\kappa^{3}\right) d x
\end{array}\right.
$$

First, when $\gamma$ is not a circle, we show that (4.3) has at most one solution. If both $(v, \lambda)$ and $(\tilde{v}, \tilde{\lambda})$ satisfy $(4.3)$, then we have

$$
\left\{\begin{array}{l}
-V^{\prime \prime}+\kappa^{2} V=-\Lambda \kappa \\
L \Lambda=-\int_{0}^{L} \kappa V d x
\end{array}\right.
$$

where $V:=v-\tilde{v}$ and $\Lambda:=\lambda-\tilde{\lambda}$. Integrating by part, we obtain

$$
\int_{0}^{L}\left\{\left(V^{\prime}\right)^{2}+\kappa^{2} V^{2}\right\} d x=\frac{1}{L}\left\{\int_{0}^{L} \kappa V d x\right\}^{2} \leq \int_{0}^{L} \kappa^{2} V^{2} d x .
$$

Hence $V$ is a constant, so that $V \equiv V_{0}$. By (4.4),

$$
\kappa^{2} V_{0}=\frac{1}{L} \kappa V_{0} \int_{0}^{L} \kappa d x=\frac{2 \pi n}{L} \kappa V_{0},
$$

where $n:=(1 / 2 \pi) \int_{0}^{L} \kappa d x$ denotes the rotation number of $\gamma$. (Note that the rotation number $n$ is invariant. See Lemma 5.1.) If $V_{0} \neq 0$, then $\kappa=0$ or $\kappa=2 \pi n / L$. Since $\gamma$ is neither a straight line nor a circle, these relations are not possible. Therefore $V_{0}=0$, so that $v=\tilde{v}$. This implies $\lambda=\tilde{\lambda}$.

Next, we shall prove the existence of a solution of (4.3). Let $u(x, t)$ and $w(x, t)$ be the solutions of the equations

$$
-u^{\prime \prime}+\kappa^{2} u=\kappa^{4}-\left(\kappa^{\prime}\right)^{2}, \quad-w^{\prime \prime}+\kappa^{2} w=-\kappa .
$$

Note that by Lemma $2.1 \kappa$ determines $u$ and $w$ uniquely. Clearly $v=u+\lambda w$ satisfies (4.3) if and only if

$$
\left(L+\int_{0}^{L} \kappa w d x\right) \lambda=\int_{0}^{L}\left(-\kappa u+\kappa^{3}\right) d x .
$$


Therefore, if $L+\int_{0}^{L} \kappa w d x \neq 0, \lambda$ is determined by (4.6). From the equation for $w$, it holds that

$$
\int_{0}^{L}\left\{\left(w^{\prime}\right)^{2}+\kappa^{2} w^{2}\right\} d x=-\int_{0}^{L} \kappa w d x \leq L^{\frac{1}{2}}\left\{\int_{0}^{L}\left\{\left(w^{\prime}\right)^{2}+\kappa^{2} w^{2}\right\} d x\right\}^{\frac{1}{2}} .
$$

Hence we see that $L+\int_{0}^{L} \kappa w d x \geq 0$. If $L+\int_{0}^{L} \kappa w d x=0$, then $\int_{0}^{L}\left\{\left(w^{\prime}\right)^{2}+\kappa^{2} w^{2}\right\} d x=$ $\int_{0}^{L} \kappa^{2} w^{2} d x$. Thus we see that $w$ is a constant function. Then, by $(4.5), \kappa(x)(\kappa(x) w+1)=$ 0 . If $\kappa\left(x_{0}\right)=0$ at some point $x_{0}$, then $\kappa(x)=0$ in a neighborhood of $x_{0}$, because $\kappa(x) w+1 \neq 0$ for $x$ close to $x_{0}$. Hence the set $\{x \mid \kappa(x)=0\}$ is open. On the other hand, this set is closed, because $\kappa(x)$ is continuous. Therefore, it holds either $\kappa(x) \neq 0$ for any $x \in S_{L}^{1}$ or $\kappa(x) \equiv 0$. But, if $\kappa(x) \equiv 0$, then $\gamma$ is a straight line, which is not possible. Thus we see that $\kappa(x) \neq 0$ for any $x \in S_{L}^{1}$. Consequently, we have $\kappa(x) w+1 \equiv 0$, so that $\kappa(x) \equiv-1 / w$. Since $\gamma(x, t)$ is a closed curve, $\gamma(x, t)$ must be a circle. Then, by (4.5), we have $u=\kappa^{2}$, and hence $v=\kappa^{2}-(\lambda / \kappa)$. Then (4.6) holds for any $\lambda \in \mathbb{R}$, because the right-hand side of (4.6) is equal to 0 . Therefore, in this case, the set of the solutions is given by (4.2). If $L+\int_{0}^{L} \kappa w d x>0$, then $\lambda(t)$ is given by

$$
\lambda=\frac{\int_{0}^{L}\left(-\kappa u+\kappa^{3}\right) d x}{L+\int_{0}^{L} \kappa w d x} .
$$

Thus the proof is completed.

To construct a mild solution of (GT), we reduce (GT) to an integral equation. For this purpose, we define a closed linear operator $A=\left(\partial^{4} / \partial x^{4}\right)+1$ on $L^{p}\left(S_{L}^{1}\right)$ with domain $\mathcal{D}(A)=W^{4, p}\left(S_{L}^{1}\right)$, where $p \geq 3$. (The reason to choose $p \geq 3$ will become clear in the proof of Lemma 4.11.) It is easy to check that $-A$ generates the analytic semigroup $e^{-t A}$ on $L^{p}\left(S_{L}^{1}\right)$. Let $\gamma=(\varphi, \psi)$. Then $(\mathrm{GT})$ becomes as follows:

$$
\left\{\begin{array}{l}
\frac{\partial \varphi}{\partial t}=-A \varphi+f(\varphi, \psi, v, \lambda), \\
\frac{\partial \psi}{\partial t}=-A \psi+g(\varphi, \psi, v, \lambda), \\
-\frac{\partial^{2} v}{\partial x^{2}}+\left|\frac{\partial^{2} \gamma}{\partial x^{2}}\right|^{2} v=2\left|\frac{\partial^{2} \gamma}{\partial x^{2}}\right|^{4}-\left|\frac{\partial^{3} \gamma}{\partial x^{3}}\right|^{2}-\lambda \boldsymbol{n} \cdot \frac{\partial^{2} \gamma}{\partial x^{2}}, \\
L \lambda=-\int_{0}^{L}\left\{\left(\boldsymbol{n} \cdot \frac{\partial^{2} \gamma}{\partial x^{2}}\right) v-\left(\boldsymbol{n} \cdot \frac{\partial^{2} \gamma}{\partial x^{2}}\right)^{3}\right\} d x,
\end{array}\right.
$$

where $f$ and $g$ are given by

$$
\begin{aligned}
& f(\varphi, \psi, v, \lambda)=\frac{\partial}{\partial x}\left\{\left(v-2\left|\frac{\partial^{2} \gamma}{\partial x^{2}}\right|^{2}\right) \frac{\partial \varphi}{\partial x}\right\}-\lambda \frac{\partial \psi}{\partial x}+\varphi \\
& g(\varphi, \psi, v, \lambda)=\frac{\partial}{\partial x}\left\{\left(v-2\left|\frac{\partial^{2} \gamma}{\partial x^{2}}\right|^{2}\right) \frac{\partial \psi}{\partial x}\right\}+\lambda \frac{\partial \varphi}{\partial x}+\psi
\end{aligned}
$$

We choose $\alpha$ such that $3 / 4+1 /(4 p)<\alpha<7 / 8$ and define the fractional power $A^{\alpha}$. Let $X^{\alpha}:=\mathcal{D}\left(A^{\alpha}\right)$. Since $A$ has a bounded inverse, we can define the norm in $X^{\alpha}$ by $\|u\|_{(\alpha)}:=\left\|A^{\alpha} u\right\|_{L^{p}}$. Since $3 / 4<\alpha$, it holds that $X^{\alpha} \subset W^{3, p}\left(S_{L}^{1}\right)$, so that there exists a 
positive constant $C(\alpha)$ such that $\|\cdot\|_{W^{3, p}} \leq C(\alpha)\|\cdot\|_{(\alpha)}$. (For the proof of these standard facts, see [3] and [12].)

We now assume that the initial closed curve $\gamma_{0}=\left(\varphi_{0}, \psi_{0}\right)$ is not a circle, and $\left(\varphi_{0}, \psi_{0}\right) \in$ $(\mathcal{D}(A))^{2}$. Then, by Lemma 4.1, we can define $v_{0}$ and $\lambda_{0}$ as the solutions of

$$
\left\{\begin{array}{l}
-v^{\prime \prime}+\left|\gamma_{0}^{\prime \prime}\right|^{2} v=2\left|\gamma_{0}^{\prime \prime}\right|^{4}-\left|\gamma_{0}^{(3)}\right|^{2}-\lambda \boldsymbol{n}_{0} \cdot \gamma_{0}^{\prime \prime} \\
L \lambda=-\int_{0}^{L}\left\{\left(\boldsymbol{n}_{0} \cdot \gamma_{0}^{\prime \prime}\right) v-\left(\boldsymbol{n}_{0} \cdot \gamma_{0}^{\prime \prime}\right)^{3}\right\} d x
\end{array}\right.
$$

Moreover, from the assumption on $\gamma_{0}$, there exists a positive constant $\delta_{0}$ such that

$$
L+\int_{0}^{L}\left(\boldsymbol{n}_{0} \cdot \gamma_{0}^{\prime \prime}\right) w_{0} d x \geq \delta_{0}
$$

where $w_{0}$ is the solution of $-w^{\prime \prime}+\left|\gamma_{0}^{\prime \prime}\right|^{2} w=-\boldsymbol{n}_{0} \cdot \gamma_{0}^{\prime \prime}$. To construct a mild solution of (GT), we define $\gamma_{j}, v_{j}$, and $\lambda_{j}$ inductively as follows:

$$
\left\{\begin{array}{l}
\varphi_{j+1}=e^{-t A} \varphi_{0}+\int_{0}^{t} e^{-(t-s) A} f\left(\varphi_{j}(s), \psi_{j}(s), v_{j}(s), \lambda_{j}(s)\right) d s \\
\psi_{j+1}=e^{-t A} \psi_{0}+\int_{0}^{t} e^{-(t-s) A} g\left(\varphi_{j}(s), \psi_{j}(s), v_{j}(s), \lambda_{j}(s)\right) d s \\
\text { where } v_{j} \text { and } \lambda_{j} \text { are the solutions of } \\
-v^{\prime \prime}+\left|\gamma_{j}^{\prime \prime}\right|^{2} v=2\left|\gamma_{j}^{\prime \prime}\right|^{4}-\left|\gamma_{j}^{(3)}\right|^{2}-\lambda \boldsymbol{n}_{j} \cdot \gamma_{j}^{\prime \prime}, \\
L \lambda=-\int_{0}^{L}\left\{\left(\boldsymbol{n}_{j} \cdot \gamma_{j}^{\prime \prime}\right) v-\left(\boldsymbol{n}_{j} \cdot \gamma_{j}^{\prime \prime}\right)^{3}\right\} d x
\end{array}\right.
$$

To prove that $\gamma_{j}, v_{j}$ and $\lambda_{j}$ are defined by (SA) for all $j$, we prepare several lemmas. Let us set $f_{j}(\cdot):=f\left(\varphi_{j}(\cdot), \psi_{j}(\cdot), v_{j}(\cdot), \lambda_{j}(\cdot)\right)$ and $g_{j}(\cdot):=g\left(\varphi_{j}(\cdot), \psi_{j}(\cdot), v_{j}(\cdot), \lambda_{j}(\cdot)\right)$ for short.

Lemma 4.2. Choose $\varepsilon>0$ such that $\left\|\gamma_{0}{ }^{\prime \prime}\right\|_{L^{2}}-2 C(\alpha) L^{(p-2) / 2 p} \varepsilon>0$. For this $\varepsilon$, suppose that $\left\|\varphi_{j}-\varphi_{0}\right\|_{(\alpha)} \leq \varepsilon$ and $\left\|\psi_{j}-\psi_{0}\right\|_{(\alpha)} \leq \varepsilon$. Then there exists a positive constant $K_{1}$ depending only on $\left\|\varphi_{0}\right\|_{(\alpha)},\left\|\psi_{0}\right\|_{(\alpha)}$ and $\varepsilon$ such that

$$
\left|\int_{0}^{L} \boldsymbol{n}_{j} \cdot \frac{\partial^{2} \gamma_{j}}{\partial x^{2}} w_{j} d x-\int_{0}^{L} \boldsymbol{n}_{0} \cdot \frac{\partial^{2} \gamma_{0}}{\partial x^{2}} w_{0} d x\right| \leq K_{1} \varepsilon .
$$

Proof. Note that

$$
\begin{aligned}
& \int_{0}^{L} \mid \boldsymbol{n}_{j} \cdot \gamma_{j}^{\prime \prime} w_{j}-\boldsymbol{n}_{0} \cdot \gamma_{0}^{\prime \prime} w_{0} \mid d x \\
& \leq \sup _{x \in S_{L}^{1}}\left|w_{0}\right|\left\|\boldsymbol{n}_{j} \cdot \gamma_{j}^{\prime \prime}-\boldsymbol{n}_{0} \cdot \gamma_{0}^{\prime \prime}\right\|_{L^{1}}+\sup _{x \in S_{L}^{1}}\left|w_{j}-w_{0}\right|\left\|\boldsymbol{n}_{j} \cdot \gamma_{j}{ }^{\prime \prime}\right\|_{L^{1}}
\end{aligned}
$$

The right-hand side of (4.9) is estimated as follows. First, we have

$$
\begin{aligned}
\left\|\boldsymbol{n}_{j} \cdot \gamma_{j}{ }^{\prime \prime}-\boldsymbol{n}_{0} \cdot \gamma_{0}{ }^{\prime \prime}\right\|_{L^{1}} \leq & \left\|\psi_{j}{ }^{\prime}\right\|_{L^{2}}\left\|\varphi_{j}{ }^{\prime \prime}-\varphi_{0}{ }^{\prime \prime}\right\|_{L^{2}}+\left\|\varphi_{0}{ }^{\prime \prime}\right\|_{L^{2}}\left\|\psi_{j}{ }^{\prime}-\psi_{0}{ }^{\prime}\right\|_{L^{2}} \\
& +\left\|\varphi_{j}{ }^{\prime}\right\|_{L^{2}}\left\|\psi_{j}^{\prime \prime}-\psi_{0}{ }^{\prime \prime}\right\|_{L^{2}}+\left\|\psi_{0}{ }^{\prime \prime}\right\|_{L^{2}}\left\|\varphi_{j}{ }^{\prime}-\varphi_{0}{ }^{\prime}\right\|_{L^{2}} \\
\leq & C\left(\left\|\varphi_{0}\right\|_{(\alpha)}+\left\|\psi_{0}\right\|_{(\alpha)}+\varepsilon\right) \varepsilon .
\end{aligned}
$$


THE MOTION OF ELASTIC CLOSED CURVES UNDER THE AREA-PRESERVING CONDITION 11

We estimate $\sup _{x}\left|w_{j}-w_{0}\right|$. Since $w_{j}-w_{0}$ satisfies

$$
-\left(w_{j}-w_{0}\right)^{\prime \prime}+\left|\gamma_{j}^{\prime \prime}\right|^{2}\left(w_{j}-w_{0}\right)=-w_{0}\left(\left|\gamma_{j}^{\prime \prime}\right|^{2}-\left|\gamma_{0}^{\prime \prime}\right|^{2}\right)-\left(\boldsymbol{n}_{j} \cdot \gamma_{j}^{\prime \prime}-\boldsymbol{n}_{0} \cdot \gamma_{0}^{\prime \prime}\right),
$$

by Lemma 2.1, we get

$$
\begin{aligned}
& \sup _{x \in S_{L}^{1}}\left|w_{j}-w_{0}\right| \\
& \quad \leq 2\left(L+\left\|\gamma_{j}^{\prime \prime}\right\|_{L^{2}}^{-2}\right)\left\{\sup _{x \in S_{L}^{1}}\left|w_{0}\right|\left\|\left|\gamma_{j}^{\prime \prime}\right|^{2}-\left|\gamma_{0}^{\prime \prime}\right|^{2}\right\|_{L^{1}}+\left\|\boldsymbol{n}_{j} \cdot \gamma_{j}^{\prime \prime}-\boldsymbol{n}_{0} \cdot \gamma_{0}^{\prime \prime}\right\|_{L^{1}}\right\} .
\end{aligned}
$$

But, we have

$$
\begin{aligned}
& \left\|\left|\gamma_{j}{ }^{\prime \prime}\right|^{2}-\left|\gamma_{0}{ }^{\prime \prime}\right|^{2}\right\|_{L^{1}} \leq\left\|\varphi_{j}^{\prime \prime}-\varphi_{0}^{\prime \prime}\right\|_{L^{2}}^{2}+\left\|\psi_{j}{ }^{\prime \prime}-\psi_{0}{ }^{\prime \prime}\right\|_{L^{2}}^{2} \leq C \varepsilon \\
& \left\|\gamma_{j}^{\prime \prime}-\gamma_{0}{ }^{\prime \prime}\right\|_{L^{2}} \leq\left\|\varphi_{j}{ }^{\prime \prime}-\varphi_{0}{ }^{\prime \prime}\right\|_{L^{2}}+\left\|\psi_{j}{ }^{\prime \prime}-\psi_{0}{ }^{\prime \prime}\right\|_{L^{2}} \leq 2 C(\alpha) L^{(p-2) / 2 p} \varepsilon
\end{aligned}
$$

By the assumption on $\varepsilon$, it holds that

$$
\left\|\gamma_{j}^{\prime \prime}\right\|_{L^{2}} \geq\left\|\gamma_{0}^{\prime \prime}\right\|_{L^{2}}-2 C(\alpha) L^{(p-2) / 2 p} \varepsilon>0
$$

By these relations, we see that there exists a positive constant $C$ depending only on $\left\|\varphi_{0}\right\|_{(\alpha)},\left\|\psi_{0}\right\|_{(\alpha)}$ and $\varepsilon$ such that $\sup _{x \in S_{L}^{1}}\left|w_{j}-w_{0}\right| \leq C \varepsilon$. Consequently, we obtain (4.8).

Suppose that $\gamma_{j}$ is defined by (SA). Then, by Lemma 4.1, $\lambda_{j}$ is given by

$$
\lambda_{j}=\frac{\int_{0}^{L}\left\{-\boldsymbol{n}_{j} \cdot \gamma_{j}^{\prime \prime} u_{j}+\left(\boldsymbol{n}_{j} \cdot \gamma_{j}^{\prime \prime}\right)^{3}\right\} d x}{L+\int_{0}^{L} \boldsymbol{n}_{j} \cdot \gamma_{j}^{\prime \prime} w_{j} d x}
$$

unless the denominator of (4.10) vanishes. Here $u_{j}$ and $w_{j}$ are the solutions of the equations

$$
-u^{\prime \prime}+\left|\gamma_{j}^{\prime \prime}\right|^{2} u=2\left|\gamma_{j}^{\prime \prime}\right|^{4}-\left|\gamma_{j}{ }^{(3)}\right|^{2},-w^{\prime \prime}+\left|\gamma_{j}^{\prime \prime}\right|^{2} w=-\boldsymbol{n}_{j} \cdot \gamma_{j}^{\prime \prime} .
$$

Fix $\varepsilon_{1}>0$ and $\varepsilon_{2}>0$ such that

$$
\left\|\gamma_{0}{ }^{\prime \prime}\right\|_{L^{2}}-2 C(\alpha) L^{(p-2) / 2 p} \varepsilon_{1}>0, \quad \delta_{0}-K_{1} \varepsilon_{2}>\frac{\delta_{0}}{2} .
$$

Let us put $\varepsilon_{*}:=\min \left\{\varepsilon_{1}, \varepsilon_{2}\right\}$. By Lemma 4.2 , we see that

$$
\left|L+\int_{0}^{L} \boldsymbol{n}_{j} \cdot \gamma_{j}^{\prime \prime} w_{j} d x\right| \geq \frac{\delta_{0}}{2}
$$

whenever $\max \left\{\left\|\varphi_{j}-\varphi_{0}\right\|_{(\alpha)},\left\|\psi_{j}-\psi_{0}\right\|_{(\alpha)}\right\} \leq \varepsilon_{*}$. Therefore $\gamma_{j+1}$ is well defined for this $\varepsilon_{*}$. 
Lemma 4.3. Suppose $\max \left\{\left\|\varphi_{j}-\varphi_{0}\right\|_{(\alpha)},\left\|\psi_{j}-\psi_{0}\right\|_{(\alpha)}\right\} \leq \varepsilon_{*}$. Then there exist positive constants $K_{2}, K_{3}, K_{4}, K_{5}$ and $K_{6}$ depending only on $\left\|\varphi_{0}\right\|_{(\alpha)},\left\|\psi_{0}\right\|_{(\alpha)}$ and $\varepsilon_{*}$ such that

$$
\begin{aligned}
& \left|\lambda_{j}(t)-\lambda_{0}\right| \leq K_{2} \varepsilon_{*}, \\
& \sup _{x \in S_{L}^{1}}\left|v_{j}(x, t)-v_{0}(x)\right| \leq K_{3} \varepsilon_{*}, \\
& \sup _{x \in S_{L}^{1}}\left|v_{j}^{\prime}(x, t)-v_{0}^{\prime}(x)\right| \leq K_{4} \varepsilon_{*}, \\
& \left\|f_{j}-f_{0}\right\|_{L^{p}} \leq K_{5} \varepsilon_{*}, \\
& \left\|g_{j}-g_{0}\right\|_{L^{p}} \leq K_{6} \varepsilon_{*} .
\end{aligned}
$$

Proof. First, we prove (4.11). By the assumption on $\varepsilon_{*}$, we have

$$
\left|\lambda_{j}(t)-\lambda_{0}\right| \leq\left(4 / \delta_{0}^{2}\right)\left(L I_{1}+\left|I_{2}\right|\right)
$$

where

$$
\begin{aligned}
I_{1}:= & \left\|-\boldsymbol{n}_{j} \cdot \gamma_{j}^{\prime \prime} u_{j}+\left(\boldsymbol{n}_{j} \cdot \gamma_{j}^{\prime \prime}\right)^{3}+\boldsymbol{n}_{0} \cdot \gamma_{0}{ }^{\prime \prime} u_{0}-\left(\boldsymbol{n}_{0} \cdot \gamma_{0}{ }^{\prime \prime}\right)^{3}\right\|_{L^{1}}, \\
I_{2}:= & \left(\int_{0}^{L} \boldsymbol{n}_{0} \cdot \gamma_{0}^{\prime \prime} w_{0} d x\right)\left(\int_{0}^{L}\left\{-\boldsymbol{n}_{j} \cdot \gamma_{j}^{\prime \prime} u_{j}+\left(\boldsymbol{n}_{j} \cdot \gamma_{j}^{\prime \prime}\right)^{3}\right\} d x\right) \\
& \quad-\left(\int_{0}^{L} \boldsymbol{n}_{j} \cdot \gamma_{j}^{\prime \prime} w_{j} d x\right)\left(\int_{0}^{L}\left\{-\boldsymbol{n}_{0} \cdot \gamma_{0}{ }^{\prime \prime} u_{0}+\left(\boldsymbol{n}_{0} \cdot \gamma_{0}^{\prime \prime}\right)^{3}\right\} d x\right) .
\end{aligned}
$$

By the same arguments as in the proof of Lemma 4.2, we have $\max \left\{I_{1},\left|I_{2}\right|\right\} \leq C \varepsilon_{*}$, where $C$ is a positive constant depending only on $\left\|\varphi_{0}\right\|_{(\alpha)},\left\|\psi_{0}\right\|_{(\alpha)}$, and $\varepsilon_{*}$. Therefore we obtain (4.11). Next we turn to (4.12). Since $v_{j}-v_{0}=\left(u_{j}-u_{0}\right)+\lambda_{j}\left(w_{j}-w_{0}\right)+w_{0}\left(\lambda_{j}-\lambda_{0}\right)$, we obtain (4.12) by (4.11) and the estimates for $\sup _{x}\left|u_{j}-u_{0}\right|$ and $\sup _{x}\left|w_{j}-w_{0}\right|$. By virtue of Lemma 2.1, we get

$$
\max \left\{\sup _{x \in S_{L}^{1}}\left|u_{j}{ }^{\prime}-u_{0}{ }^{\prime}\right|, \sup _{x \in S_{L}^{1}}\left|w_{j}^{\prime}-w_{0}{ }^{\prime}\right|\right\} \leq C\left(\left\|\varphi_{0}\right\|_{(\alpha)},\left\|\psi_{0}\right\|_{(\alpha)}, \varepsilon_{*}\right) \varepsilon_{*} .
$$

Therefore we obtain (4.13) along the same line as in the derivation of (4.12). Similarly, it is easy to check that (4.14) and (4.15) hold.

Let us put $B:=\max \left\{\left\|f_{0}\right\|_{L^{p}},\left\|g_{0}\right\|_{L^{p}}\right\}, M:=\max \left\{K_{5}, K_{6}\right\}$. For $u \in X^{\alpha}$, it holds that

$$
\begin{gathered}
\left\|A^{\alpha} e^{-t A} u\right\|_{L^{p}} \leq \frac{C_{\alpha}}{t^{\alpha}}\|u\|_{L^{p}} \quad(\alpha \geq 0, \quad t>0), \\
\left\|\left(e^{-t A}-I\right) u\right\|_{L^{p}} \leq \frac{C_{1-\alpha}}{\alpha} t^{\alpha}\left\|A^{\alpha} u\right\|_{L^{p}} \quad(0<\alpha \leq 1) .
\end{gathered}
$$

(For the proof of these estimates, see [3].) We shall often use these estimates in the following arguments.

Lemma 4.4. Let $T$ be a positive number such that

$$
\left\{\begin{array}{l}
\max \left\{\left\|e^{-t A} \varphi_{0}-\varphi_{0}\right\|_{(\alpha)},\left\|e^{-t A} \psi_{0}-\psi_{0}\right\|_{(\alpha)}\right\} \leq \frac{\varepsilon_{*}}{2} \quad \text { for } \quad 0 \leq t \leq T, \\
\frac{C_{\alpha}}{1-\alpha}\left(M \varepsilon_{*}+B\right) T^{1-\alpha} \leq \frac{\varepsilon_{*}}{2} .
\end{array}\right.
$$

Let $\varphi_{j}, \psi_{j}, v_{j}$, and $\lambda_{j}$ be defined by (SA). Then it holds that $\varphi_{j} \in C\left([0, T] ; X^{\alpha}\right)$ and $\psi_{j} \in C\left([0, T] ; X^{\alpha}\right)$ and $\max \left\{\left\|\varphi_{j}-\varphi_{0}\right\|_{(\alpha)},\left\|\psi_{j}-\psi_{0}\right\|_{(\alpha)}\right\} \leq \varepsilon_{*}$ for any positive integer $j$. 
Proof. We shall prove the assertion by induction. Suppose that the conclusion holds for $0 \leq n \leq j$. By virtue of Lemmas 4.2 and 4.3 , it is sufficient to prove $\left(\varphi_{j+1}, \psi_{j+1}\right) \in$ $\left(C\left([0, T] ; X^{\alpha}\right)\right)^{2}$ and $\left\|\varphi_{j+1}-\varphi_{0}\right\|_{(\alpha)} \leq \varepsilon_{*}$ and $\left\|\psi_{j+1}-\psi_{0}\right\|_{(\alpha)} \leq \varepsilon_{*}$. First, we show that $\varphi_{j+1} \in X^{\alpha}$. For this purpose, it is sufficient to check that

$$
F(t):=\int_{0}^{t} e^{-(t-s) A} f_{j}(s) d s \in X^{\alpha}
$$

For $\rho>0$, let us define

$$
F_{\rho}(t)= \begin{cases}\int_{0}^{t-\rho} e^{-(t-s) A} f_{j}(s) d s & \text { for } \quad \rho \leq t \leq T \\ 0 & \text { for } t<\rho .\end{cases}
$$

By Lemma 4.3, we have

$$
\left\|F_{\rho}(t)-F(t)\right\|_{L^{p}} \leq \int_{t-\rho}^{t}\left\|e^{-(t-s) A}\right\|\left\|f_{j}(s)\right\|_{L^{p}} d s \leq C\left(M \varepsilon_{*}+B\right) \rho .
$$

Therefore $F_{\rho}$ converges to $F$ as $\rho \rightarrow 0$ uniformly on $[\delta, T]$ for any $\delta \in(0, T)$. Moreover, we can check that $A^{\alpha} F_{\rho}(t)=\int_{0}^{t-\rho} A^{\alpha} e^{-(t-s) A} f_{j}(s) d s$. Hence we get

$$
\left\|A^{\alpha} F_{\rho}(t)-\int_{0}^{t} A^{\alpha} e^{-(t-s) A} f_{j}(s) d s\right\|_{L^{p}} \leq \frac{C_{\alpha}}{1-\alpha}\left(M \varepsilon_{*}+B\right) \rho^{1-\alpha} .
$$

The right-hand side of (4.17) converges to 0 as $\rho \rightarrow 0$ uniformly on $[\delta, T]$ for any $0<\delta<T$. Since $A^{\alpha}$ is a closed operator, it holds that

$$
F(t) \in X^{\alpha}, \quad A^{\alpha} F(t)=\int_{0}^{t} A^{\alpha} e^{-(t-s) A} f_{j}(s) d s .
$$

Therefore, $\varphi_{j+1} \in X^{\alpha}$. In the same way, we can prove $\psi_{j+1} \in X^{\alpha}$. Moreover, it is easy to check that $\left(\varphi_{j+1}, \psi_{j+1}\right) \in\left(C\left([0, T] ; X^{\alpha}\right)\right)^{2}$. Next, we prove the continuity of $v_{j}$ and $\lambda_{j}$ in $t$. By replacing $\gamma_{0}, v_{0}$, and $\lambda_{0}$ in the proof of Lemma 4.3 with $\gamma_{j}(s), v_{j}(s)$, and $\lambda_{j}(s)$, respectively, we see that there exists a positive constant $C$ depending only on $\left\|\varphi_{0}\right\|_{(\alpha)},\left\|\psi_{0}\right\|_{(\alpha)}$, and $\varepsilon_{*}$ such that

$$
\begin{aligned}
& \max \left\{\left|\lambda_{j}(t)-\lambda_{j}(s)\right|, \sup _{x \in S_{L}^{1}} \mid v_{j}(x, t)\right.\left.-v_{j}(x, s)\left|, \sup _{x \in S_{L}^{1}}\right| v_{j}{ }^{\prime}(x, t)-v_{j}{ }^{\prime}(x, s) \mid\right\} \\
& \leq C\left(\left\|\varphi_{j}(t)-\varphi_{j}(s)\right\|_{(\alpha)}+\left\|\psi_{j}(t)-\psi_{j}(s)\right\|_{(\alpha)}\right) .
\end{aligned}
$$

Therefore the continuity of $\varphi_{j}$ and $\psi_{j}$ in $t$ implies the continuity of $v_{j}, v_{j}{ }^{\prime}$, and $\lambda_{j}$ in $t$. Finally, we show that $\max \left\{\left\|\varphi_{j+1}-\varphi_{0}\right\|_{(\alpha)},\left\|\psi_{j+1}-\psi_{0}\right\|_{(\alpha)}\right\} \leq \varepsilon_{*}$. By the assumption (4.16), we have

$$
\begin{aligned}
& \left\|\varphi_{j+1}-\varphi_{0}\right\|_{(\alpha)} \\
& \quad \leq\left\|e^{-t A} \varphi_{0}-\varphi_{0}\right\|_{(\alpha)}+\left\|\int_{0}^{t} e^{-(t-s) A}\left\{f_{j}(s)-f_{0}\right\} d s\right\|_{(\alpha)}+\left\|\int_{0}^{t} e^{-(t-s) A} f_{0} d s\right\|_{(\alpha)} \\
& \quad \leq \frac{\varepsilon_{*}}{2}+\int_{0}^{t} C_{\alpha}(t-s)^{-\alpha}\left\|f_{j}(s)-f_{0}\right\|_{L^{p}} d s+\int_{0}^{t} C_{\alpha}(t-s)^{-\alpha}\left\|f_{0}\right\|_{L^{p}} d s \\
& \quad \leq \frac{\varepsilon_{*}}{2}+\frac{C_{\alpha}}{1-\alpha}\left(M \varepsilon_{*}+B\right) t^{1-\alpha} \leq \varepsilon_{*} .
\end{aligned}
$$


Similarly, we can prove $\left\|\psi_{j+1}-\psi_{0}\right\|_{(\alpha)} \leq \varepsilon_{*}$. The proof of Lemma 4.4 is now completed.

Let us set

$$
\begin{aligned}
\Phi\left(\varphi_{j}, \psi_{j}\right) & =e^{-t A} \varphi_{0}+\int_{0}^{t} e^{-(t-s) A} f_{j}(s) d s, \\
\Psi\left(\varphi_{j}, \psi_{j}\right) & =e^{-t A} \psi_{0}+\int_{0}^{t} e^{-(t-s) A} g_{j}(s) d s, \\
S_{\varepsilon_{*}, 1} & =\left\{\eta \in C\left([0, T] ; X^{\alpha}\right) \mid\left\|\eta-\varphi_{0}\right\|_{(\alpha)} \leq \varepsilon_{*}\right\}, \\
S_{\varepsilon_{*}, 2} & =\left\{\eta \in C\left([0, T] ; X^{\alpha}\right) \mid\left\|\eta-\psi_{0}\right\|_{(\alpha)} \leq \varepsilon_{*}\right\} .
\end{aligned}
$$

Let us define the norm $\|u\|_{T}:=\sup _{t \in[0, T]}\|u(t)\|_{(\alpha)}$. Then, $\left(S_{\varepsilon_{*}, 1},\|\cdot\|_{T}\right)$ and $\left(S_{\varepsilon_{*}, 2},\|\cdot\|_{T}\right)$ are Banach spaces. We define a map $\mathcal{F}: S_{\varepsilon_{*}, 1} \times S_{\varepsilon_{*}, 2} \rightarrow S_{\varepsilon_{*}, 1} \times S_{\varepsilon_{*}, 2}$ as follows:

$$
\mathcal{F}\left(\varphi_{j}, \psi_{j}\right)=\left(\Phi\left(\varphi_{j}, \psi_{j}\right), \Psi\left(\varphi_{j}, \psi_{j}\right)\right) .
$$

We shall prove that $\mathcal{F}$ is a contraction map. To do this, we make use of the following lemma.

Lemma 4.5. For any positive integers $i$ and $j$, there are positive constants $L_{1}$ and $L_{2}$ independent of $i$ and $j$ such that

$$
\begin{aligned}
& \left\|f_{i}-f_{j}\right\|_{L^{p}} \leq L_{1}\left(\left\|\varphi_{i}-\varphi_{j}\right\|_{(\alpha)}+\left\|\psi_{i}-\psi_{j}\right\|_{(\alpha)}\right), \\
& \left\|g_{i}-g_{j}\right\|_{L^{p}} \leq L_{2}\left(\left\|\varphi_{i}-\varphi_{j}\right\|_{(\alpha)}+\left\|\psi_{i}-\psi_{j}\right\|_{(\alpha)}\right) .
\end{aligned}
$$

Proof. By replacing $\gamma_{0}, v_{0}$, and $\lambda_{0}$ in the proof of Lemma 4.3 with $\gamma_{i}, v_{i}$, and $\lambda_{i}$ respectively, we obtain

(4.20) $\max \left\{\left|\lambda_{i}-\lambda_{j}\right|, \sup _{x \in S_{L}^{1}}\left|v_{i}-v_{j}\right|, \sup _{x \in S_{L}^{1}}\left|v_{i}{ }^{\prime}-v_{j}{ }^{\prime}\right|\right\} \leq C\left(\left\|\varphi_{i}-\varphi_{j}\right\|_{(\alpha)}+\left\|\psi_{i}-\psi_{j}\right\|_{(\alpha)}\right)$

where the positive constant $C$ depends only on $\left\|\varphi_{0}\right\|_{(\alpha)},\left\|\psi_{0}\right\|_{(\alpha)}$, and $\varepsilon_{*}$. Thus we derive (4.18) and (4.19) in the same way as in the proof of Lemma 4.3.

We are now in a position to prove that $\mathcal{F}$ is a contraction map.

Lemma 4.6. For a positive constant $T$ satisfying $(4.16)$ and $\frac{C_{\alpha}}{1-\alpha} T^{1-\alpha}\left(L_{1}+L_{2}\right)<1, \mathcal{F}$ is a contraction map from $S_{\varepsilon_{*}, 1} \times S_{\varepsilon_{*}, 2}$ into itself.

Proof. For any $i$ and $j$, by virtue of Lemma 4.5, we have

$$
\begin{aligned}
\left\|\Phi\left(\varphi_{i}, \psi_{i}\right)-\Phi\left(\varphi_{j}, \psi_{j}\right)\right\|_{(\alpha)} & \leq \frac{C_{\alpha}}{1-\alpha} T^{1-\alpha} L_{1}\left(\left\|\varphi_{i}-\varphi_{j}\right\|_{T}+\left\|\psi_{i}-\psi_{j}\right\|_{T}\right), \\
\left\|\Psi\left(\varphi_{i}, \psi_{i}\right)-\Psi\left(\varphi_{j}, \psi_{j}\right)\right\|_{(\alpha)} & \leq \frac{C_{\alpha}}{1-\alpha} T^{1-\alpha} L_{2}\left(\left\|\varphi_{i}-\varphi_{j}\right\|_{T}+\left\|\psi_{i}-\psi_{j}\right\|_{T}\right) .
\end{aligned}
$$

By the assumption on $T$, we conclude that $\mathcal{F}$ is a contraction map.

By these lemmas, we can construct a mild solution of (GT) as follows: 
Lemma 4.7. Suppose that $T$ satisfies the assumption of Lemma 4.6. For $\varphi_{j}, \psi_{j}, v_{j}$, and $\lambda_{j}$ determined by $(\mathrm{SA})$, there exist $\varphi \in S_{\varepsilon_{*}, 1}, \psi \in S_{\varepsilon_{*}, 2}, v \in C\left([0, T] ; W^{2,2}\left(S_{L}^{1}\right)\right)$, and $\lambda \in C([0, T] ; \mathbb{R})$ such that

$$
\left\{\begin{array}{l}
\varphi=e^{-t A} \varphi_{0}+\int_{0}^{t} e^{-(t-s) A} f(\varphi(s), \psi(s), v(s), \lambda(s)) d s, \\
\psi=e^{-t A} \psi_{0}+\int_{0}^{t} e^{-(t-s) A} g(\varphi(s), \psi(s), v(s), \lambda(s)) d s, \\
-v^{\prime \prime}+\left|\gamma^{\prime \prime}\right|^{2} v=2\left|\gamma^{\prime \prime}\right|^{4}-\left|\gamma^{(3)}\right|^{2}-\lambda \boldsymbol{n} \cdot \gamma^{\prime \prime}, \\
L \lambda=-\int_{0}^{L}\left\{\left(\boldsymbol{n} \cdot \gamma^{\prime \prime}\right) v-\left(\boldsymbol{n} \cdot \gamma^{\prime \prime}\right)^{3}\right\} d x .
\end{array}\right.
$$

Proof. By virtue of Lemma 4.6, using the contraction mapping principle, we deduce that there exists a unique fixed point of $\mathcal{F}: \varphi \in S_{\varepsilon_{*}, 1}, \psi \in S_{\varepsilon_{*}, 2}$. We shall prove the convergence of $v_{j}$ and $\lambda_{j}$. Let us define $\|v\|_{W^{2,2}, T}:=\sup _{t \in[0, T]}\|v(\cdot, t)\|_{W^{2,2}}$ and $\|\lambda\|_{C, T}:=$ $\sup _{t \in[0, T]}|\lambda(t)|$. Then spaces $\left(C\left([0, T] ; W^{2,2}\left(S_{L}^{1}\right)\right),\|\cdot\|_{W^{2,2}, T}\right)$ and $\left(C([0, T] ; \mathbb{R}),\|\cdot\|_{C, T}\right)$ are Banach spaces. By (4.20) in the proof of Lemma 4.5 and the equation for $v_{j},\left\{v_{j}\right\}_{j}$ and $\left\{\lambda_{j}\right\}_{j}$ are Cauchy sequences in $\left(C\left([0, T] ; W^{2,2}\left(S_{L}^{1}\right)\right),\|\cdot\|_{W^{2,2}, T}\right)$ and $\left(C([0, T] ; \mathbb{R}),\|\cdot\|_{C, T}\right)$, respectively. Hence, there exist $v \in C\left([0, T] ; W^{2,2}\left(S_{L}^{1}\right)\right)$ and $\lambda \in C([0, T] ; \mathbb{R})$ such that $v_{j} \rightarrow v, \lambda_{j} \rightarrow \lambda$ as $j \rightarrow \infty$. Then it is easy to check that $\varphi, \psi, v$, and $\lambda$ satisfy (GT-I).

Up to now, we have constructed a mild solution of (GT). Next we show that this mild solution is actually a strong solution of $(\mathrm{GT})$. We prove this assertion as follows:

Lemma 4.8. $f(\varphi, \psi, v, \lambda)$ and $g(\varphi, \psi, v, \lambda)$ are locally Hölder continuous functions from $(0, T)$ to $L^{p}\left(S_{L}^{1}\right)$.

Lemma 4.9. $\varphi$ and $\psi$ belong to $C\left([0, T] ; X^{\alpha}\right) \cap C^{1}\left([0, T] ; L^{p}\right)$ and satisfy $(\mathrm{GT})$.

Since these arguments are standard, we omit the proof of Lemmas 4.8 and 4.9. Next we show that $(\varphi, \psi)$ is indeed a classical solution of $(\mathrm{GT})$. To prove the continuity of $\partial \varphi / \partial t$ and $\partial \psi / \partial t$, we make use of the following lemma.

Lemma 4.10 (D. Henry [3]). Let $X$ be a Banach space and $A$ be a sectorial operator on $X$. Suppose that $u:[0, T) \rightarrow X$ satisfies $\|u(t)-u(s)\| \leq V(s)(t-s)^{\mu}$ for $0<s<t<T<\infty$ and $0<\mu \leq 1$, where $V(\cdot)$ is continuous on $(0, T)$ and satisfies $\int_{0}^{T} V(s) d s<\infty$. Then there exist $0 \leq \nu<\mu$ and a positive constant $C$ being independent of $\mu, \nu$, and $u(\cdot)$ such that

$$
U(t):=\int_{0}^{t} e^{-(t-s) A} u(s) d s
$$

is a continuously differentiable function from $(0, T)$ into $\mathcal{D}\left(A^{\nu}\right)$ and satisfies

$$
\left\|\frac{d U}{d t}(t)\right\|_{(\nu)} \leq C t^{-\nu}\|u\|+C \int_{0}^{t}(t-s)^{\mu-\nu-1} V(s) d s \quad \text { for } \quad 0<t<T .
$$

Moreover, $(d / d t) U(t):(0, T) \rightarrow X^{\nu}$ is a locally Hölder continuous function.

We omit the proof. Using this lemma, we shall prove the following:

Lemma 4.11. $\varphi$ and $\psi$ are classical solutions of (GT). 
Proof. Let $\omega(t):=C\left(t^{-1+\zeta+\alpha}+t^{-\alpha}+t^{1-\alpha}\right)$ with $\alpha<\zeta<1$. Then it is easy to check that

$$
\|f(t+h)-f(t)\|_{L^{p}},\|g(t+h)-g(t)\|_{L^{p}} \leq \omega(t) h^{\beta}
$$

with $0<\beta<1-\alpha$. Here we choose $\beta$ close to $1-\alpha$. Let us set

$$
J_{1}=\int_{0}^{t} e^{-(t-s) A} f(s) d s, \quad J_{2}=\int_{0}^{t} e^{-(t-s) A} g(s) d s .
$$

By using Lemma 4.10, we see that $\partial J_{1} / \partial t$ and $\partial J_{2} / \partial t$ are locally Hölder continuous functions from $(0, T)$ into $X^{\theta}$ for any $0 \leq \theta<\beta<1-\alpha<1 / 4-1 /(4 p)$. Since $p \geq 3$, we can choose $\theta$ such that $1 / 8<\theta<\beta$. Then, we can check that $\partial \varphi / \partial t$ and $\partial \psi / \partial t$ are locally Hölder continuous functions from $(0, T)$ into $X^{\theta}$ with respect to $t$. Moreover, since $\theta>1 / 8$, it holds that $X^{\theta} \subset C^{\delta}\left(S_{L}^{1}\right)$ with $0<\delta<(1 / 2)-(1 / p)$. Therefore $\partial \varphi / \partial t$ and $\partial \psi / \partial t$ are also locally Hölder continuous on $S_{L}^{1}$. With these preparations, we can deduce the conclusion of the lemma by a standard argument.

We shall prove that the solution $\gamma(x, t)$ satisfies the inextensibility condition $(\mathrm{C} 1)$. The following lemma holds for any initial closed curve $\gamma_{0}$ with $\left|\gamma_{0}{ }^{\prime}(x)\right| \equiv 1$.

Lemma 4.12. Let $\gamma_{0}(x)$ be any $C^{\infty}$ closed curve with $\left|\gamma_{0}^{\prime}(x)\right| \equiv 1$. Then the solution $\gamma(x, t)$ of $(\mathrm{GT})$ satisfies $\left|\gamma^{\prime}(x, t)\right| \equiv 1$.

Proof. First we have

$$
\frac{\partial}{\partial t}\left(\left|\gamma^{\prime}\right|^{2}\right)=2 \gamma^{\prime} \cdot\left\{-\gamma^{(5)}+\left(\left(v-2\left|\gamma^{\prime \prime}\right|^{2}\right) \gamma^{\prime}\right)^{\prime \prime}+\lambda R \gamma^{\prime \prime}\right\} .
$$

By the second equation of (GT), (4.21) becomes

$$
\frac{\partial}{\partial t}\left(\left|\gamma^{\prime}\right|^{2}\right)=-\left(\left|\gamma^{\prime}\right|^{2}\right)^{(4)}+\sum_{i=1}^{2} P_{i}\left(\left|\gamma^{\prime}\right|-1\right)^{(i)}
$$

where $P_{0}, P_{1}$ and $P_{2}$ are bounded functions and defined by

$$
P_{0}=2\left(v^{\prime \prime}-4\left|\gamma^{(3)}\right|^{2}-4 \gamma^{\prime \prime} \cdot \gamma^{(4)}\right), P_{1}=2\left(v^{\prime}-4 \gamma^{\prime \prime} \cdot \gamma^{(3)}\right), P_{2}=v-2\left|\gamma^{\prime \prime}\right|^{2} .
$$

Then, we can proceed in the same way as in [5].

\section{LONG TIME EXISTENCE}

We shall prove that (GT) has a solution $\gamma(x, t)$ for all $t>0$. In this section, we assume that $\gamma_{0}$ satisfies

$$
\left|\mathcal{A}_{0}\right|<\frac{L^{2}}{4 \pi}
$$

where $L^{2} / 4 \pi$ is the area of a circle with perimeter $L$. By the isoperimetric inequality, $\gamma_{0}(x)$ is not a circle whenever (5.1) holds. Let $\gamma(x, t)$ be the solution of $(\mathrm{GT})$ on $[0, T)$, where $T>0$. First, we prepare several lemmas.

Lemma 5.1. The center of gravity of $\gamma(x, t)$ is invariant. 
Proof. The center of gravity of $\gamma$ is given by $\frac{1}{L} \int_{0}^{L} \gamma d x$. Since

$$
\frac{d}{d t} \int_{0}^{L} \gamma d x=\int_{0}^{L}\left\{-\gamma^{(4)}+\left(\left(v-2\left|\gamma^{\prime \prime}\right|^{2}\right) \gamma^{\prime}\right)^{\prime}+\lambda \boldsymbol{n}\right\} d x=0,
$$

we obtain the conclusion.

Without loss of generality, we may assume that the center of gravity of $\gamma_{0}(x)$ is $(0,0)$.

Lemma 5.2. The rotation number of $\gamma(x, t)$ is invariant.

Proof. We have proved that (GT) with an initial data $\gamma_{0}(x)$ has a unique classical solution $(\gamma(x, t), v(x, t), \lambda(t))$ for $t \in[0, T)$. Therefore $\gamma(x, t)$ is regularly homotopic to $\gamma_{0}(x)$. This implies that the rotation number of $\gamma(x, t)$ is equal to that of $\gamma_{0}(x)$ for any $t \in[0, T)$.

Now we suppose that the rotation number of $\gamma_{0}(x)$ is 1 , so that $\int_{0}^{L} \kappa_{0} d x=2 \pi$. Then, by virtue of Lemma $5.2, \int_{0}^{L} \kappa d x=2 \pi$. Next we shall derive an estimate of the elastic energy $\left\|\gamma^{\prime \prime}\right\|_{L^{2}}^{2}$. Since $\gamma^{\prime \prime}=\kappa \boldsymbol{n}$, it holds that $\left\|\gamma^{\prime \prime}\right\|_{L^{2}}=\|\kappa\|_{L^{2}}$.

Lemma 5.3. $\|\kappa\|_{L^{2}}$ is non-increasing. Moreover it holds that

$$
\|\kappa\|_{L^{2}}^{2} \geq \frac{4 \pi^{2}}{L}
$$

where the equality holds if and only if $\kappa=2 \pi / L$ a.e.

Proof. Since

$$
\frac{1}{2} \frac{d}{d t}\|\kappa\|_{L^{2}}^{2}=\left\langle\gamma^{(4)}, \dot{\gamma}\right\rangle_{L^{2}}=\left\langle\gamma^{(4)}-\left\{\left(v-2\left|\gamma^{\prime \prime}\right|^{2}\right) \gamma^{\prime}\right\}^{\prime}-\lambda \boldsymbol{n}, \dot{\gamma}\right\rangle_{L^{2}}=-\|\dot{\gamma}\|_{L^{2}}^{2},
$$

$\|\kappa\|_{L^{2}}$ is non-increasing. To prove (5.2), we consider the strain energy $\int_{0}^{L}\left(\kappa-\frac{2 \pi}{L}\right)^{2} d x$, where $2 \pi / L$ is the curvature of a circle with perimeter $L$. By Lemma 5.2 , we have

$$
0 \leq \int_{0}^{L}\left(\kappa-\frac{2 \pi}{L}\right)^{2} d x=\int_{0}^{L} \kappa^{2} d x-\frac{4 \pi^{2}}{L} .
$$

Hence we obtain (5.2). Moreover, if $\int_{0}^{L} \kappa^{2} d x=4 \pi^{2} / L$ holds, then $\int_{0}^{L}\left(\kappa-\frac{2 \pi}{L}\right)^{2} d x=0$, and hence it holds that $\kappa=2 \pi / L$ a.e.

If $\gamma(x, t)$ is not a circle, by virtue of Lemma $4.1, \lambda(t)$ is expressed as (4.7). To derive the estimate of $\lambda(t)$, we make use of the following lemma.

Lemma 5.4. Suppose that $\gamma$ is a solution of $(\mathrm{GT})$ on $[0, T)$ for some $T>0$. Then, it holds that

$$
\inf _{t \in[0, T)}\left(L+\int_{0}^{L} \kappa w d x\right)>0 .
$$

Proof. Otherwise, there would exist $\left\{t_{j}\right\}_{j=1}^{\infty} \subset[0, T)$ such that $L+\int_{0}^{L} \kappa_{j} w_{j} d x \rightarrow 0$ as $j \rightarrow \infty$, where $\kappa_{j}(\cdot):=\kappa\left(\cdot, t_{j}\right), w_{j}(\cdot):=w\left(\cdot, t_{j}\right)$. Since $w_{j}$ satisfies $-w_{j}^{\prime \prime}+\kappa_{j}{ }^{2} w_{j}=-\kappa_{j}$, we have

$$
\int_{0}^{L} \kappa_{j} w_{j} d x=-\int_{0}^{L}\left\{\left(w_{j}^{\prime}\right)^{2}+\kappa_{j}^{2} w_{j}^{2}\right\} d x
$$


By the assumption,

$$
\int_{0}^{L}\left\{\left(w_{j}^{\prime}\right)^{2}+\kappa_{j}^{2} w_{j}^{2}\right\} d x \longrightarrow L \text { as } \quad j \longrightarrow \infty .
$$

Moreover, by (5.4),

$$
\begin{aligned}
\int_{0}^{L}\left\{\left(w_{j}^{\prime}\right)^{2}+\kappa_{j}{ }^{2} w_{j}{ }^{2}\right\} d x & \leq L^{1 / 2}\left\{\int_{0}^{L} \kappa_{j}{ }^{2} w_{j}{ }^{2} d x\right\}^{1 / 2} \\
& \leq L^{1 / 2}\left\{\int_{0}^{L}\left\{\left(w_{j}^{\prime}\right)^{2}+\kappa_{j}{ }^{2} w_{j}{ }^{2}\right\} d x\right\}^{1 / 2}
\end{aligned}
$$

and hence we see $\int_{0}^{L} \kappa_{j}^{2} w_{j}^{2} d x \rightarrow L$ as $j \rightarrow \infty$. Then, by (5.5), we deduce that

$$
\int_{0}^{L}\left(w_{j}^{\prime}\right)^{2} d x \longrightarrow 0 \text { as } j \longrightarrow \infty \text {. }
$$

By Lemmas 2.1 and 5.3, we have $\sup _{x \in S_{L}^{1}}\left|w_{j}(x)\right| \leq\left(2 L+\left\|\kappa_{j}\right\|_{L^{2}}^{-2}\right)\left\|\kappa_{j}\right\|_{L^{1}} \leq C_{\gamma_{0}}$. Therefore $\left\{w_{j}\right\}$ is uniformly bounded. Similarly, we obtain $\sup _{x \in S_{L}^{1}}\left|w_{j}{ }^{\prime}(x)\right| \leq C_{\gamma_{0}}$. For any $x, y \in S_{L}^{1}$, it holds that

$$
\left|w_{j}(x)-w_{j}(y)\right|=\left|\int_{y}^{x} w_{j}^{\prime}(\sigma) d \sigma\right| \leq \sqrt{|x-y|}\left\|w_{j}^{\prime}\right\|_{L^{2}} .
$$

Hence we deduce that $\left\{w_{j}\right\}$ is equicontinuous. By the Ascoli-Arzelá theorem, there exists $\left\{w_{j_{n}}\right\} \subset\left\{w_{j}\right\}$ such that $w_{j_{n}}$ converges to $w_{\infty}$ as $n \rightarrow \infty$ uniformly on $S_{L}^{1}$. Furthermore, by (5.6) and (5.7), we have $\left|w_{\infty}(x)-w_{\infty}(y)\right|=0$ for any $x, y \in S_{L}^{1}$. Therefore $w_{\infty}$ is a constant.

Next, we compute the value of $w_{\infty}$. By Lemma 5.3, $\left\{\kappa_{j}\right\}$ is bounded in $L^{2}\left(S_{L}^{1}\right)$. Thus, $\left\{\kappa_{j}\right\}$ contains a subsequence which is still denoted by $\left\{\kappa_{j}\right\}$ for simplicity such that $\kappa_{j} \rightarrow$ $\kappa_{\infty}$ weakly in $L^{2}\left(S_{L}^{1}\right)$. By Lemma 5.2 , we have

$$
2 \pi=\int_{0}^{L} \kappa_{j} d x=\left\langle\kappa_{j}, 1\right\rangle_{L^{2}} \longrightarrow\left\langle\kappa_{\infty}, 1\right\rangle_{L^{2}}=\int_{0}^{L} \kappa_{\infty} d x .
$$

Moreover, we get

$$
\begin{aligned}
& \left|\int_{0}^{L}\left\{\kappa_{j} w_{j}-\kappa_{\infty} w_{\infty}\right\} d x\right| \\
& \quad \leq\left|\int_{0}^{L} \kappa_{j}\left(w_{j}-w_{\infty}\right) d x\right|+\left|\int_{0}^{L} w_{\infty}\left(\kappa_{j}-\kappa_{\infty}\right) d x\right| \longrightarrow 0 \text { as } \quad j \longrightarrow \infty,
\end{aligned}
$$

and hence $\int_{0}^{L} \kappa_{\infty} w_{\infty} d x=-L$. By (5.8), we find that

$$
w_{\infty}=-\frac{L}{2 \pi} .
$$

Next, we consider the limit function $\kappa_{\infty}$. First, we prove that $\int_{0}^{L} \kappa_{j}{ }^{2} d x \rightarrow 4 \pi^{2} / L$ as $j \rightarrow \infty$. Since $-w_{j}{ }^{\prime \prime}+\kappa_{j}{ }^{2} w_{j}=-\kappa_{j}$ and $\int_{0}^{L} \kappa_{j} d x=2 \pi$, we have $\int_{0}^{L} \kappa_{j}{ }^{2} w_{j} d x=-2 \pi$. Hence we obtain $\int_{0}^{L} \kappa_{j}{ }^{2} w_{\infty} d x \rightarrow-2 \pi$ as $j \rightarrow \infty$. Combining this with (5.10), we have

$$
\int_{0}^{L} \kappa_{j}^{2} d x \longrightarrow \frac{4 \pi^{2}}{L} \quad \text { as } \quad j \longrightarrow \infty
$$


Thus, by (5.11) and the definition of $\kappa_{\infty}$, we can check that

$$
\left\|\kappa_{\infty}\right\|_{L^{2}}^{2} \leq \frac{4 \pi^{2}}{L}
$$

By Lemma 5.3 and (5.12), we deduce that $\int_{0}^{L} \kappa_{\infty}^{2} d x=4 \pi^{2} / L$, so that $\kappa_{\infty}=2 \pi / L$ a.e.

Finally, we prove that $\gamma_{j}$ converges to a curve $\gamma_{\infty}$ whose curvature is $\kappa_{\infty}$. Put $\gamma_{j}=$ $\left(\varphi_{j}, \psi_{j}\right)$. Let $\theta_{j}$ be the angle between the tangent $\gamma_{j}^{\prime}$ and the $x_{1}$-axis. Suppose $\gamma_{j}(0)=$ $(0,0)$ and $\theta_{j}(0)=0, \theta_{j}(L)=2 \pi$. Then it holds that $\varphi_{j}(x)=\int_{0}^{x} \cos \theta_{j}(\sigma) d \sigma, \psi_{j}(x)=$ $\int_{0}^{x} \sin \theta_{j}(\sigma) d \sigma, \theta_{j}(x)=\int_{0}^{x} \kappa_{j}(\sigma) d \sigma$. By Lemma 5.3, it is easy to check that $\left\{\theta_{j}\right\}$ is uniformly bounded and equicontinuous. Therefore, by the Ascoli-Arzelá theorem, we see that there exists $\left\{\theta_{j_{n}}\right\} \subset\left\{\theta_{j}\right\}$ such that $\theta_{j_{n}}$ converges to $\theta_{\infty}$ as $n \rightarrow \infty$ uniformly on $S_{L}^{1}$. In what follows, we write $\theta_{j}$ instead of $\theta_{j_{n}}$ for simplicity. On the other hand, it holds that

$$
\theta_{j}(x)=\left\langle\kappa_{j}, I_{[0, x]}\right\rangle_{L^{2}} \longrightarrow\left\langle\kappa_{\infty}, I_{[0, x]}\right\rangle_{L^{2}}=\int_{0}^{x} \kappa_{\infty} d \sigma,
$$

where $I_{[0, x]}$ is the characteristic function of the interval $[0, x]$. Hence we have $\theta_{j} \rightarrow \theta_{\infty}=$ $\int_{0}^{x} \kappa_{\infty} d \sigma$. Since $\kappa_{\infty}=2 \pi / L$ a.e., it holds that $\theta_{\infty}(x)=2 \pi x / L$. We easily see that $\varphi_{j}$ and $\psi_{j}$ converge to $\varphi_{\infty}=(L / 2 \pi) \sin (2 \pi x / L)$ and $\psi_{\infty}=-(L / 2 \pi) \cos (2 \pi x / L)+L / 2 \pi$ uniformly on $S_{L}^{1}$, respectively. Moreover, $\varphi_{j}{ }^{\prime}(x)$ and $\psi_{j}{ }^{\prime}(x)$ converge to $\cos \theta_{\infty}(x)$ and $\sin \theta_{\infty}(x)$ uniformly on $S_{L}^{1}$, respectively. Therefore we obtain

$$
\mathcal{A}\left(\gamma_{j}\right)=-\frac{1}{2} \int_{0}^{L} \boldsymbol{n}_{j} \cdot \gamma_{j} d x \longrightarrow-\frac{1}{2} \int_{0}^{L} \boldsymbol{n}_{\infty} \cdot \gamma_{\infty} d x=\frac{L^{2}}{4 \pi} .
$$

This contradicts the fact that the area enclosed by $\gamma(x, t)$ is preserved, and the proof is now complete.

By virtue of Lemma 5.4, we can estimate $\lambda(t), v(x, t)$ and $v^{\prime}(x, t)$ as follows:

Lemma 5.5. There exists a positive constant $C$ such that

$$
\max \left\{|\lambda|, \sup _{x \in S_{L}^{1}}|v|, \sup _{x \in S_{L}^{1}}\left|\frac{\partial v}{\partial x}\right|\right\} \leq C\left(1+\left\|\frac{\partial^{3} \gamma}{\partial x^{3}}\right\|_{L^{2}}^{2}\right) .
$$

Proof. First, we estimate $\lambda$. By Lemma 5.4, there exists a positive constant $\delta_{1}$ such that $L+\int_{0}^{L} \kappa w d x \geq \delta_{1}$ for any $t \in[0, T)$. Then we have

$$
|\lambda| \leq \delta_{1}^{-1} \int_{0}^{L}\left\{\left|\left(\boldsymbol{n} \cdot \gamma^{\prime \prime}\right) u\right|+\left|\boldsymbol{n} \cdot \gamma^{\prime \prime}\right|^{3}\right\} d x \leq C\left(\sup _{x \in S_{L}^{1}}|u|\left\|\gamma^{\prime \prime}\right\|_{L^{2}}+\sup _{x \in S_{L}^{1}}\left|\gamma^{\prime \prime}\right|\left\|\gamma^{\prime \prime}\right\|_{L^{2}}^{2}\right) .
$$

Here, by Lemmas 2.1, 2.3 and 5.3, we have

$$
\begin{aligned}
& \sup _{x \in S_{L}^{1}}\left|\gamma^{\prime \prime}\right| \leq \sqrt{2}\left\|\gamma^{\prime \prime}\right\|_{L^{2}}^{\frac{1}{2}}\left\|\gamma^{(3)}\right\|_{L^{2}}^{\frac{1}{2}} \leq C_{\gamma_{0}}\left\|\gamma^{(3)}\right\|_{L^{2}}^{\frac{1}{2}}, \\
& \sup _{x \in S_{L}^{1}}|u| \leq\left(2 L+\left\|\gamma^{\prime \prime}\right\|_{L^{2}}^{-2}\right)\left\|2\left|\gamma^{\prime \prime}\right|^{4}-\left|\gamma^{(3)}\right|^{2}\right\|_{L^{1}} \leq C_{\gamma_{0}}\left(1+\left\|\gamma^{(3)}\right\|_{L^{2}}^{2}\right) .
\end{aligned}
$$

Combining these three inequalities gives us an estimate for $\lambda$. Second we proceed to estimate $v$. By the proof of Lemma 5.4, we know that $\sup _{x \in S_{L}^{1}}|w| \leq C_{\gamma_{0}}$. Since $v=u+\lambda w$, we obtain the estimate for $v$. We have also seen that $\sup _{x \in S_{L}^{1}}\left|w^{\prime}\right| \leq C_{\gamma_{0}}$. By Lemmas 2.1, 
2.3 and 5.3, we can prove that $\sup _{x \in S_{L}^{1}}\left|u^{\prime}\right| \leq C\left(1+\left\|\gamma^{(3)}\right\|_{L^{2}}^{2}\right)$. Since $v^{\prime}=u^{\prime}+\lambda w^{\prime}$, we obtain (5.13).

To prove that (GT) has a solution for all $t>0$, we make use of the following lemma. Let us put $H^{m}:=W^{m, 2}$ for short.

Lemma 5.6. Suppose that $\gamma(x, t)$ is a classical solution of (GT) and satisfies $\left\|\gamma^{(3)}\right\|_{L^{2}} \leq$ $C_{1}$ on $[0, T]$ for some $T>0$. Then, for any integer $n>3$, there exists a positive constant $C_{2}$ depending only on $C_{1}, \gamma_{0}, n$ and $T$ such that $\left\|\gamma^{(n)}\right\|_{L^{2}} \leq C_{2}$ on $[0, T]$.

Proof. We prove the assertion by induction. Suppose that the conclusion holds for $n>3$, so that $\left\|\gamma^{(n)}\right\|_{L^{2}}$ is bounded on $[0, T]$. Then,

$$
\begin{aligned}
\frac{1}{2} \frac{d}{d t}\left\|\gamma^{(n+1)}\right\|_{L^{2}}^{2} & =\left\langle\gamma^{(n+1)}, \dot{\gamma}^{(n+1)}\right\rangle_{L^{2}} \\
& =\left\langle\gamma^{(n+3)},-\gamma^{(n+3)}+\left(\left(v-2\left|\gamma^{\prime \prime}\right|^{2}\right) \gamma^{\prime}\right)^{(n)}+\lambda \boldsymbol{n}^{(n-1)}\right\rangle_{L^{2}} \\
& \leq-\frac{1}{2}\left\|\gamma^{(n+3)}\right\|_{L^{2}}^{2}+\frac{1}{2}\left\|\left(\left(v-2\left|\gamma^{\prime \prime}\right|^{2}\right) \gamma^{\prime}\right)^{(n)}+\lambda \boldsymbol{n}^{(n-1)}\right\|_{L^{2}}^{2}
\end{aligned}
$$

We shall estimate the second term of the right-hand side of (5.14). First, by Lemma 5.5,

$$
\left\|\lambda \boldsymbol{n}^{(n-1)}\right\|_{L^{2}}^{2} \leq C\left(1+\left\|\gamma^{(3)}\right\|_{L^{2}}^{2}\right)\left\|\gamma^{(n)}\right\|_{L^{2}}^{2} \leq C .
$$

Second we estimate $\left\|\left(v \gamma^{\prime}\right)^{(n)}\right\|_{L^{2}}$. By Lemma 2.2, we get $\|v\|_{C^{n-2}} \leq C$. Thus we have

$$
\begin{aligned}
\left\|\left(v \gamma^{\prime}\right)^{(n)}\right\|_{L^{2}} & \leq C\left(1+\left\|v^{(n)} \gamma^{\prime}\right\|_{L^{2}}+\left\|v^{(n-1)} \gamma^{\prime \prime}\right\|_{L^{2}}+\left\|v \gamma^{(n+1)}\right\|_{L^{2}}\right) \\
& \leq C\left(1+\|v\|_{H^{n}}+\left\|\gamma^{(n+1)}\right\|_{L^{2}}\right) .
\end{aligned}
$$

Hence it is sufficient to estimate $\|v\|_{H^{n}}$ and $\left\|\gamma^{(n+1)}\right\|_{L^{2}}$. For $i=1,2$, it holds that

$$
\begin{aligned}
\left\|\gamma^{(n+i)}\right\|_{L^{2}} \leq C\left\|\gamma^{(n)}\right\|_{L^{2}}^{(3-i) / 3}\left\|\gamma^{(n+3)}\right\|_{L^{2}}^{i / 3} \leq C\left\|\gamma^{(n+3)}\right\|_{L^{2}}^{i / 3}, \\
\sup _{x \in S_{L}^{1}}\left|\gamma^{(2+i)}\right| \leq C\left\|\gamma^{(3+i)}\right\|_{L^{2}} \leq C\left\|\gamma^{(n+i)}\right\|_{L^{2}} \leq C\left\|\gamma^{(n+3)}\right\|_{L^{2}}^{i / 3} .
\end{aligned}
$$

By these inequalities and Lemma 2.2, we have

$$
\begin{aligned}
\|v\|_{H^{n}} & \leq C\left(1+\left\|\left|\gamma^{\prime \prime}\right|^{2}\right\|_{H^{n-2}}^{N}\right)\left\|2\left|\gamma^{\prime \prime}\right|^{4}-\left|\gamma^{(3)}\right|^{2}-\lambda \boldsymbol{n} \cdot \gamma^{\prime \prime}\right\|_{H^{n-2}} \\
& \leq C\left(1+\left\|\left|\gamma^{(3)}\right|^{2}\right\|_{H^{n-2}}\right) \\
& \leq C\left(1+\sup _{x \in S_{L}^{1}}\left|\gamma^{(3)}\right|\left\|\gamma^{(n+1)}\right\|_{L^{2}}+\sup _{x \in S_{L}^{1}}\left|\gamma^{(4)}\right|\left\|\gamma^{(n)}\right\|_{L^{2}}\right) \leq C\left(1+\left\|\gamma^{(n+3)}\right\|_{L^{2}}^{2 / 3}\right) .
\end{aligned}
$$

Therefore we obtain

$$
\left\|\left(v \gamma^{\prime}\right)^{(n)}\right\|_{L^{2}}^{2} \leq C\left(1+\left\|\gamma^{(n+3)}\right\|_{L^{2}}^{4 / 3}\right)
$$


By the same arguments, we can estimate $\left\|\left(\left|\gamma^{\prime \prime}\right|^{2} \gamma^{\prime}\right)^{(n)}\right\|_{L^{2}}$ as follows:

$$
\left\|\left(\left|\gamma^{\prime \prime}\right|^{2} \gamma^{\prime}\right)^{(n)}\right\|_{L^{2}}^{2} \leq C\left(1+\left\|\gamma^{(n+3)}\right\|_{L^{2}}^{4 / 3}\right) .
$$

Consequently,

$$
\begin{aligned}
\frac{d}{d t}\left\|\gamma^{(n+1)}\right\|_{L^{2}}^{2} & \leq-\frac{1}{2}\left\|\gamma^{(n+3)}\right\|_{L^{2}}^{2}+C\left(1+\left\|\gamma^{(n+3)}\right\|_{L^{2}}^{4 / 3}\right) \\
& \leq\left(-\frac{1}{2}+\frac{2}{3} \varepsilon^{3 / 2}\right)\left\|\gamma^{(n+3)}\right\|_{L^{2}}^{2}+C\left(1+\frac{1}{3 \varepsilon^{3}}\right)
\end{aligned}
$$

Choosing $\varepsilon$ so small that $-\frac{1}{2}+\frac{2}{3} \varepsilon^{3 / 2}<0$, we complete the proof.

By Lemma 5.6, if $\left\|\gamma^{(3)}\right\|_{L^{2}}$ is bounded on any finite time interval, then (GT) has a solution for all $t>0$. To prove that $\left\|\gamma^{(3)}\right\|_{L^{2}}$ is bounded, we prepare two lemmas.

Lemma 5.7. Suppose that $\gamma$ is a classical solution of (GT) on $[0, T)$ for some $T>0$. Then it holds that

$$
\inf _{t \in[0, T)}\left\|\gamma \cdot \frac{\partial \gamma}{\partial x}\right\|_{L^{1}}>0
$$

Proof. For contradiction, suppose that there exists $\left\{t_{j}\right\}_{j=1}^{\infty} \subset[0, T)$ such that

$$
\left\|\gamma_{j} \cdot \gamma_{j}^{\prime}\right\|_{L^{1}} \rightarrow 0 \quad \text { as } j \rightarrow \infty
$$

where $\gamma_{j}:=\gamma\left(\cdot, t_{j}\right)$. By virtue of Lemma 5.3, we have

$$
\left\|\left(\left|\gamma_{j}\right|^{2}\right)^{\prime \prime}\right\|_{L^{2}} \leq C\left(1+\sup _{x \in S_{L}^{1}}\left|\gamma_{j}\right|\left\|\gamma_{j}^{\prime \prime}\right\|_{L^{2}}\right) \leq C_{\gamma_{0}} .
$$

By (5.16), we deduce that $\left\{\gamma_{j} \cdot \gamma_{j}{ }^{\prime}\right\}_{j}$ is uniformly bounded and equicontinuous. By the Ascoli-Arzelá theorem, there exists $\left\{\gamma_{j_{n}}\right\}_{j_{n}} \subset\left\{\gamma_{j}\right\}_{j}$ such that $\gamma_{j_{n}} \cdot \gamma_{j_{n}}{ }^{\prime}$ converges to 0 as $n \rightarrow \infty$ uniformly on $S_{L}^{1}$. Therefore, since $\left(\left|\gamma_{j_{n}}\right|^{2}\right)^{\prime}=2 \gamma_{j_{n}} \cdot \gamma_{j_{n}}{ }^{\prime}$, there exists a constant $C$ such that $\left|\gamma_{j_{n}}\right|$ converges to $C$ as $n \rightarrow \infty$ uniformly on $S_{L}^{1}$. This implies that $\gamma_{j_{n}}$ converges to a circle, which contradicts $\mathcal{A} \equiv \mathcal{A}_{0}<L^{2} / 4 \pi$. Hence the proof is now complete.

Lemma 5.8. Suppose that $\gamma$ is a classical solution of (GT) on $[0, T)$ for some $T>0$. Then there exist positive constants $C_{1}$ and $C_{2}$ depending only on $\gamma_{0}$ and $T$ such that

$$
\frac{d}{d t}\left\|\frac{\partial^{2} \gamma}{\partial x^{2}}\right\|_{L^{2}}^{2} \leq-C_{1}\left\|\frac{\partial^{4} \gamma}{\partial x^{4}}\right\|_{L^{2}}^{2}+C_{2} .
$$

Proof. In the proof of Lemma 5.3, we verified that

$$
\frac{d}{d t}\left\|\gamma^{\prime \prime}\right\|_{L^{2}}^{2}=-\|\dot{\gamma}\|_{L^{2}}^{2}
$$

Since

$$
\dot{\gamma} \cdot \gamma^{\prime}=v^{\prime}-\frac{1}{2}\left(\left|\gamma^{\prime \prime}\right|^{2}\right)^{\prime}
$$

the normal direction $(\dot{\gamma})_{N}$ of $\dot{\gamma}$ is expressed as follows:

$$
(\dot{\gamma})_{N}=-\gamma^{(4)}+\left(v-2\left|\gamma^{\prime \prime}\right|^{2}\right) \gamma^{\prime \prime}-\frac{3}{2}\left(\left|\gamma^{\prime \prime}\right|^{2}\right)^{\prime} \gamma^{\prime}+\lambda \boldsymbol{n} .
$$


Let us set $\Gamma:=-\gamma^{(3)}+\left(v-2\left|\gamma^{\prime \prime}\right|^{2}\right) \gamma^{\prime}+\lambda R \gamma$, so that $\Gamma^{\prime}=\dot{\gamma}$. Since

$$
\Gamma \cdot \gamma^{\prime}=v-\left|\gamma^{\prime \prime}\right|^{2}-\lambda \gamma \cdot \boldsymbol{n}
$$

the normal direction $(\Gamma)_{N}$ of $\Gamma$ is given by

$$
(\Gamma)_{N}=-\gamma^{(3)}-\left|\gamma^{\prime \prime}\right|^{2} \gamma^{\prime}+\lambda\left(\gamma \cdot \gamma^{\prime}\right) \boldsymbol{n}
$$

From (5.19) and Lemma 5.1, it follows that

$$
\begin{aligned}
\left|\int_{0}^{L} \Gamma d x\right| & =\left|\int_{0}^{L}\left\{\left(v-2\left|\gamma^{\prime \prime}\right|^{2}\right) \gamma^{\prime}\right\} d x\right| \\
& \leq\left|\int_{0}^{L}\left\{\left(v-\frac{1}{2}\left|\gamma^{\prime \prime}\right|^{2}\right)^{\prime} \gamma\right\} d x\right|+\frac{3}{2}\left|\int_{0}^{L}\left\{\left(\left|\gamma^{\prime \prime}\right|^{2}\right)^{\prime} \gamma\right\} d x\right| \\
& =\left|\int_{0}^{L}\left(\dot{\gamma} \cdot \gamma^{\prime}\right) \gamma d x\right|+\left.\frac{3}{2}\left|\int_{0}^{L}\right| \gamma^{\prime \prime}\right|^{2} \gamma^{\prime} d x \mid \leq C\left(1+\|\dot{\gamma}\|_{L^{2}}\right) .
\end{aligned}
$$

Hence it holds that $\sup _{x \in S_{L}^{1}}|\Gamma| \leq\left|\frac{1}{L} \int_{0}^{L} \Gamma d x\right|+\left\|\Gamma^{\prime}\right\|_{L^{1}} \leq C\left(1+\|\dot{\gamma}\|_{L^{2}}\right)$. Moreover, by (5.21) and (5.22), we obtain

$$
\begin{aligned}
& \max \left\{\left.\sup _{x \in S_{L}^{1}}|v-| \gamma^{\prime \prime}\right|^{2}-\lambda \gamma \cdot \boldsymbol{n}\left|, \quad \sup _{x \in S_{L}^{1}}\right|-\gamma^{(3)}-\left|\gamma^{\prime \prime}\right|^{2} \gamma^{\prime}+\lambda\left(\gamma \cdot \gamma^{\prime}\right) \boldsymbol{n} \mid\right\} \\
& \leq C\left(1+\|\dot{\gamma}\|_{L^{2}}\right) .
\end{aligned}
$$

It follows from (5.20) that

$$
\begin{aligned}
\|\dot{\gamma}\|_{L^{2}} & \geq\left\|(\dot{\gamma})_{N}\right\|_{L^{2}} \\
& \geq\left\|\gamma^{(4)}\right\|_{L^{2}}-\left\|\left(v-2\left|\gamma^{\prime \prime}\right|^{2}\right) \gamma^{\prime \prime}\right\|_{L^{2}}-\left\|\frac{3}{2}\left(\left|\gamma^{\prime \prime}\right|^{2}\right)^{\prime} \gamma^{\prime}\right\|_{L^{2}}-\|\lambda \boldsymbol{n}\|_{L^{2}} .
\end{aligned}
$$

We estimate the right-hand side of (5.24). By (5.23), we have

$$
|\lambda|\left\|\gamma \cdot \gamma^{\prime}\right\|_{L^{2}} \leq C\left(1+\|\dot{\gamma}\|_{L^{2}}\right)+\left\|\gamma^{(3)}\right\|_{L^{2}}+\left\|\left|\gamma^{\prime \prime}\right|^{2} \gamma^{\prime}\right\|_{L^{2}} \leq C\left(1+\|\dot{\gamma}\|_{L^{2}}+\left\|\gamma^{(4)}\right\|_{L^{2}}^{1 / 2}\right) .
$$

By Lemma 5.7, there exists a positive constant $\delta_{2}$ such that $\left\|\gamma \cdot \gamma^{\prime}\right\|_{L^{1}} \geq \delta_{2}$. Therefore we have

$$
|\lambda| \leq C \cdot L^{\frac{1}{2}} \cdot \delta_{2}^{-1}\left(1+\|\dot{\gamma}\|_{L^{2}}+\left\|\gamma^{(4)}\right\|_{L^{2}}^{1 / 2}\right) .
$$

Next, we turn to the estimate of $\left\|\left(v-2\left|\gamma^{\prime \prime}\right|^{2}\right) \gamma^{\prime \prime}\right\|_{L^{2}}$. The inequalities (5.23) and (5.25) imply

$$
\left.\sup _{x \in S_{L}^{1}}|v-| \gamma^{\prime \prime}\right|^{2}\left|\leq C\left(1+\|\dot{\gamma}\|_{L^{2}}\right)+\right| \lambda\left|\sup _{x \in S_{L}^{1}}\right| \gamma \cdot \boldsymbol{n} \mid \leq C\left(1+\|\dot{\gamma}\|_{L^{2}}+\left\|\gamma^{(4)}\right\|_{L^{2}}^{1 / 2}\right) .
$$

Hence we get

$$
\left\|\left(v-2\left|\gamma^{\prime \prime}\right|^{2}\right) \gamma^{\prime \prime}\right\|_{L^{2}} \leq\left\|\left(v-\left|\gamma^{\prime \prime}\right|^{2}\right) \gamma^{\prime \prime}\right\|_{L^{2}}+\left\|\left|\gamma^{\prime \prime}\right|^{2} \gamma^{\prime \prime}\right\|_{L^{2}} \leq C\left(1+\|\dot{\gamma}\|_{L^{2}}+\left\|\gamma^{(4)}\right\|_{L^{2}}^{1 / 2}\right) .
$$

Finally, we have

$$
\left\|\left(\left|\gamma^{\prime \prime}\right|^{2}\right)^{\prime} \gamma^{\prime}\right\|_{L^{2}} \leq 2 \sup _{x \in S_{L}^{1}}\left|\gamma^{\prime \prime}\right|\left\|\gamma^{(3)}\right\|_{L^{2}} \leq C\left\|\gamma^{(4)}\right\|_{L^{2}}^{3 / 4}
$$


THE MOTION OF ELASTIC CLOSED CURVES UNDER THE AREA-PRESERVING CONDITION 23 Then, by Young's inequality, we obtain

$$
\begin{aligned}
\|\dot{\gamma}\|_{L^{2}} & \geq\left\|\gamma^{(4)}\right\|_{L^{2}}-C\left(1+\|\dot{\gamma}\|_{L^{2}}+\left\|\gamma^{(4)}\right\|_{L^{2}}^{1 / 2}\right)-C\left\|\gamma^{(4)}\right\|_{L^{2}}^{3 / 4} \\
& \geq \frac{1}{2}\left\|\gamma^{(4)}\right\|_{L^{2}}-C\left(1+\|\dot{\gamma}\|_{L^{2}}\right)-C_{1}
\end{aligned}
$$

hence we find

$$
\|\dot{\gamma}\|_{L^{2}} \geq C_{2}\left\|\gamma^{(4)}\right\|_{L^{2}}-C_{3}
$$

Combining (5.18) with (5.26), we obtain (5.17).

By Lemmas 5.6 and 5.8, we can prove the following lemma.

Lemma 5.9. Suppose that $\gamma$ is a solution of (GT) on $[0, T)$ for some $T>0$. Then there exists a positive constant $C$ depending only on $\gamma_{0}$ and $T$ such that $\left\|\gamma^{(3)}\right\|_{L^{2}} \leq C$ on $[0, T]$.

Proof. By the Cauchy-Schwarz inequality, we have

$$
\begin{aligned}
\frac{d}{d t}\left\|\gamma^{(3)}\right\|_{L^{2}}^{2} & =2\left\langle\gamma^{(5)},-\gamma^{(5)}+\left(\left(v-2\left|\gamma^{\prime \prime}\right|^{2}\right) \gamma^{\prime}\right)^{\prime \prime}+\lambda \boldsymbol{n}^{\prime}\right\rangle_{L^{2}} \\
& \leq-\left\|\gamma^{(5)}\right\|_{L^{2}}^{2}+\left\|\left(\left(v-2\left|\gamma^{\prime \prime}\right|^{2}\right) \gamma^{\prime}\right)^{\prime \prime}+\lambda \boldsymbol{n}^{\prime}\right\|_{L^{2}}^{2}
\end{aligned}
$$

We shall estimate the right-hand side of (5.27). First, it follows from Lemmas 5.3 and 5.5 that $\left\|\lambda \boldsymbol{n}^{\prime}\right\|_{L^{2}} \leq C\left(1+\left\|\gamma^{(5)}\right\|_{L^{2}}^{2 / 3}\right)$. We recall the following interpolation inequalities:

$$
\begin{aligned}
& \left\|\gamma^{(3)}\right\|_{L^{2}} \leq\left\|\gamma^{\prime \prime}\right\|_{L^{2}}^{2 / 3}\left\|\gamma^{(5)}\right\|_{L^{2}}^{1 / 3} \leq C\left\|\gamma^{(5)}\right\|_{L^{2}}^{1 / 3} ; \\
& \left\|\gamma^{(4)}\right\|_{L^{2}} \leq\left\|\gamma^{\prime \prime}\right\|_{L^{2}}^{1 / 3}\left\|\gamma^{(5)}\right\|_{L^{2}}^{2 / 3} \leq C\left\|\gamma^{(5)}\right\|_{L^{2}}^{2 / 3} ; \\
& \sup _{x \in S_{L}^{1}}\left|\gamma^{\prime \prime}\right| \leq \sqrt{2}\left\|\gamma^{\prime \prime}\right\|_{L^{2}}^{1 / 2}\left\|\gamma^{(3)}\right\|_{L^{2}}^{1 / 2} \leq C\left\|\gamma^{(5)}\right\|_{L^{2}}^{1 / 6} ; \\
& \sup _{x \in S_{L}^{1}}\left|\gamma^{(3)}\right| \leq \sqrt{2}\left\|\gamma^{(3)}\right\|_{L^{2}}^{1 / 2}\left\|\gamma^{(4)}\right\|_{L^{2}}^{1 / 2} \leq C\left\|\gamma^{(5)}\right\|_{L^{2}}^{1 / 2}
\end{aligned}
$$

By these inequalities, we shall estimate $\left\|\left(\left(v-2\left|\gamma^{\prime \prime}\right|^{2}\right) \gamma^{\prime}\right)^{\prime \prime}\right\|_{L^{2}}$ as follows:

$$
\begin{gathered}
\left\|v^{\prime \prime} \gamma^{\prime}\right\|_{L^{2}}=\left\|v^{\prime \prime}\right\|_{L^{2}} \leq C\left(1+\left\|\gamma^{(5)}\right\|_{L^{2}}^{5 / 6}\right) ; \\
\left\|v^{\prime} \gamma^{\prime \prime}\right\|_{L^{2}} \leq C\left(1+\left\|\gamma^{(3)}\right\|_{L^{2}}^{2}\right) \leq C\left(1+\left\|\gamma^{(5)}\right\|_{L^{2}}^{2 / 3}\right) ; \\
\left\|\left(\left|\gamma^{\prime \prime}\right|^{2}\right)^{\prime} \gamma^{\prime \prime}\right\|_{L^{2}} \leq 2 \sup _{x \in S_{L}^{1}}\left|\gamma^{\prime \prime}\right|^{2}\left\|\gamma^{(3)}\right\|_{L^{2}} \leq C\left\|\gamma^{(5)}\right\|_{L^{2}}^{2 / 3} ; \\
\left\|v \gamma^{(3)}\right\|_{L^{2}} \leq C\left(1+\left\|\gamma^{(3)}\right\|_{L^{2}}^{2}\right)\left\|\gamma^{(3)}\right\|_{L^{2}} \leq C\left(1+\left\|\gamma^{(4)}\right\|_{L^{2}}\right)\left\|\gamma^{(3)}\right\|_{L^{2}} ; \\
\left\|\left|\gamma^{\prime \prime}\right|^{2} \gamma^{(3)}\right\|_{L^{2}} \leq \sup _{x \in S_{L}^{1}}\left|\gamma^{\prime \prime}\right|^{2}\left\|\gamma^{(3)}\right\|_{L^{2}} \leq C\left\|\gamma^{(5)}\right\|_{L^{2}}^{2 / 3} .
\end{gathered}
$$


Therefore we get

$$
\begin{aligned}
\frac{d}{d t}\left\|\gamma^{(3)}\right\|_{L^{2}}^{2} & \leq-\left\|\gamma^{(5)}\right\|_{L^{2}}^{2}+C\left(1+\left\|\gamma^{(5)}\right\|_{L^{2}}^{11 / 6}+\left\|\gamma^{(3)}\right\|_{L^{2}}^{2}\left\|\gamma^{(4)}\right\|_{L^{2}}^{2}\right) \\
& \leq\left(-1+\frac{11}{12} \varepsilon^{12 / 11}\right)\left\|\gamma^{(5)}\right\|_{L^{2}}^{2}+C\left(1+\frac{\varepsilon^{-12}}{12}+\left\|\gamma^{(3)}\right\|_{L^{2}}^{2}\left\|\gamma^{(4)}\right\|_{L^{2}}^{2}\right)
\end{aligned}
$$

Choosing $\varepsilon$ so small that $-1+\frac{11}{12} \varepsilon^{12 / 11}<0$, we obtain

$$
\frac{d}{d t}\left\|\gamma^{(3)}\right\|_{L^{2}}^{2} \leq C\left(1+\left\|\gamma^{(3)}\right\|_{L^{2}}^{2}\left\|\gamma^{(4)}\right\|_{L^{2}}^{2}\right)
$$

By Lemma 5.3, $\left\|\gamma^{(3)}\right\|_{L^{2}}^{2}>0$. We divide the both sides of (5.28) by $\left\|\gamma^{(3)}\right\|_{L^{2}}^{2}$ to have

$$
\frac{d}{d t} \log \left\|\gamma^{(3)}\right\|_{L^{2}}^{2} \leq C\left(1+\left\|\gamma^{(4)}\right\|_{L^{2}}^{2}\right)
$$

By using Lemma 5.8, we obtain

$$
C_{1} \frac{d}{d t} \log \left\|\gamma^{(3)}\right\|_{L^{2}}^{2}+C \frac{d}{d t}\left\|\gamma^{\prime \prime}\right\|_{L^{2}}^{2} \leq C_{3}
$$

Integrating both sides from 0 to $T$, we obtain the assertion of the lemma by virtue of Lemma 5.3.

We now come to the main result of the section.

Theorem 5.1. Let $\gamma_{0}(x)$ be a smooth closed curve with length $L$, enclosed area $\mathcal{A}_{0}$ and rotation number 1 . Suppose that $\gamma_{0}(x)$ satisfies $\left|\gamma_{0}{ }^{\prime}(x)\right| \equiv 1$ and $\left|\mathcal{A}_{0}\right|<L^{2} / 4 \pi$. Then $(\mathrm{GT})$ has a unique classical solution $(\gamma(x, t), v(x, t), \lambda(t))$ for all $t>0$.

Proof. Otherwise, there exists a positive constant $\bar{T}$ such that $\|\gamma\|_{W^{4, p}} \rightarrow \infty$ as $t \rightarrow \bar{T}$. Since $\gamma$ is a solution of (GT) on $[0, \bar{T})$, by Lemmas 5.6 and 5.9 , we see that there exists a constant $C$ such that $\left\|\gamma^{(n)}\right\|_{L^{2}} \leq C$ on $[0, \bar{T}]$ for all $n \geq 0$. This contradicts the assumption. Therefore we complete the proof.

\section{Convergence}

In this section, we shall prove that the solution $\gamma(x, t)$ converges to a stationary solution of (GT). We assume that $\gamma_{0}(x)$ is not a circle. We begin by preparing several lemmas.

Lemma 6.1. There exist positive constants $C_{1}$ and $C_{2}$ independent of $t$ such that

$$
\begin{aligned}
\max \left\{|\lambda|, \sup _{x \in S_{L}^{1}}|v|, \sup _{x \in S_{L}^{1}}\left|\frac{\partial v}{\partial x}\right|\right\} & \leq C_{1}\left(1+\left\|\frac{\partial \gamma}{\partial t}\right\|_{L^{2}}\right), \\
\sup _{x \in S_{L}^{1}}\left|\frac{\partial^{2} v}{\partial x^{2}}\right| & \leq C_{2}\left(1+\left\|\frac{\partial \gamma}{\partial t}\right\|_{L^{2}}^{3 / 2}\right) .
\end{aligned}
$$

Proof. In the proof of Lemma 5.8, we have shown (5.26). Then, by Lemma 5.5, we obtain (6.1). Moreover, by (5.26), (6.1) and the second equation of (GT), we obtain (6.2).

Lemma 6.2. For any non-negative integer $n$, there exist positive constants $C$ and $N$ independent of $t$ such that

$$
\max \left\{\|\gamma\|_{H^{n+4}},\|v\|_{H^{n+3}}\right\} \leq C\left(1+\left\|\frac{\partial \gamma}{\partial t}\right\|_{H^{n}}^{N}\right) .
$$


THE MOTION OF ELASTIC CLOSED CURVES UNDER THE AREA-PRESERVING CONDITION 25

Proof. We prove the assertion by induction. From (5.26), it follows that $\|\gamma\|_{H^{4}} \leq C(1+$ $\left.\|\dot{\gamma}\|_{L^{2}}\right)$. Then, by Lemma 2.2 , we have an estimate for $\|v\|_{H^{3}}$. Suppose that the conclusion holds for $0 \leq n \leq j$. Then,

$$
\left\|\gamma^{(j+5)}\right\|_{L^{2}} \leq\left\|\dot{\gamma}^{(j+1)}\right\|_{L^{2}}+\left\|\left(\left(v-2\left|\gamma^{\prime \prime}\right|^{2}\right) \gamma^{\prime}\right)^{(j+2)}+\lambda \boldsymbol{n}^{(j+1)}\right\|_{L^{2}} .
$$

We estimate the right-hand side of (6.4). First, we get

$$
\begin{aligned}
\left\|\left(\left|\gamma^{\prime \prime}\right|^{2} \gamma^{\prime}\right)^{(j+2)}\right\|_{L^{2}} & \leq C\left\|\gamma^{(j+4)}\right\|_{L^{2}}^{N} \leq C\left(1+\|\dot{\gamma}\|_{H^{j}}^{N_{1}}\right), \\
\left\|\left(v \gamma^{\prime}\right)^{(j+2)}\right\|_{L^{2}} & \leq C\|\gamma\|_{C^{j+3}}\|v\|_{H^{j+2}} \leq C\|\gamma\|_{H^{j+4}}\|v\|_{H^{j+2}} \leq C\left(1+\|\dot{\gamma}\|_{H^{j}}^{N}\right) .
\end{aligned}
$$

In the same way, we obtain

$$
|\lambda|\left\|\boldsymbol{n}^{(j+1)}\right\|_{L^{2}} \leq C\left(1+\|\dot{\gamma}\|_{L^{2}}\right)\left(1+\|\dot{\gamma}\|_{H^{j-2}}^{N}\right) \leq C\left(1+\|\dot{\gamma}\|_{H^{j}}^{N_{1}}\right) .
$$

Therefore, by (6.4), we have $\left\|\gamma^{(j+5)}\right\|_{L^{2}} \leq C\left(1+\|\dot{\gamma}\|_{H^{j+1}}^{N}\right)$. Similarly, by using Lemma 2.2 , we can prove the estimate of $\|v\|_{H^{j+4}}$.

Next, we shall estimate $\partial v / \partial t$ and $\partial \lambda / \partial t$. By differentiating the second and third equations of $(\mathrm{GT})$ with respect to $t$, we have

$$
\left\{\begin{array}{c}
-\frac{\partial^{2}}{\partial x^{2}} \frac{\partial v}{\partial t}+\left|\frac{\partial^{2} \gamma}{\partial x^{2}}\right|^{2} \frac{\partial v}{\partial t}=-\frac{\partial}{\partial t}\left(\left|\frac{\partial^{2} \gamma}{\partial x^{2}}\right|^{2}\right) v+2 \frac{\partial}{\partial t}\left(\left|\frac{\partial^{2} \gamma}{\partial x^{2}}\right|^{4}\right)-\frac{\partial}{\partial t}\left(\left|\frac{\partial^{3} \gamma}{\partial x^{3}}\right|^{2}\right) \\
-\lambda \frac{\partial}{\partial t}\left(\boldsymbol{n} \cdot \frac{\partial^{2} \gamma}{\partial x^{2}}\right)+\frac{\partial \lambda}{\partial t} \boldsymbol{n} \cdot \frac{\partial^{2} \gamma}{\partial x^{2}} \\
\int_{0}^{L}\left\{\frac{\partial}{\partial t}\left(\boldsymbol{n} \cdot \frac{\partial^{2} \gamma}{\partial x^{2}}\right)\left(v-3\left(\boldsymbol{n} \cdot \frac{\partial^{2} \gamma}{\partial x^{2}}\right)^{2}\right)+\left(\boldsymbol{n} \cdot \frac{\partial^{2} \gamma}{\partial x^{2}}\right) \frac{\partial v}{\partial t}+\frac{\partial \lambda}{\partial t}\right\} d x=0
\end{array}\right.
$$

Let $\partial u / \partial t$ and $\partial w / \partial t$ be the solutions of the following equations, respectively:

$$
\left\{\begin{array}{l}
-\frac{\partial^{2}}{\partial x^{2}} \frac{\partial u}{\partial t}+\left|\frac{\partial^{2} \gamma}{\partial x^{2}}\right|^{2} \frac{\partial u}{\partial t}=-\frac{\partial}{\partial t}\left(\left|\frac{\partial^{2} \gamma}{\partial x^{2}}\right|^{2}\right) u+2 \frac{\partial}{\partial t}\left(\left|\frac{\partial^{2} \gamma}{\partial x^{2}}\right|^{4}\right)-\frac{\partial}{\partial t}\left(\left|\frac{\partial^{3} \gamma}{\partial x^{3}}\right|^{2}\right) \\
-\frac{\partial^{2}}{\partial x^{2}} \frac{\partial w}{\partial t}+\left|\frac{\partial^{2} \gamma}{\partial x^{2}}\right|^{2} \frac{\partial w}{\partial t}=-\frac{\partial}{\partial t}\left(\left|\frac{\partial^{2} \gamma}{\partial x^{2}}\right|^{2}\right) w-\frac{\partial}{\partial t}\left(\boldsymbol{n} \cdot \frac{\partial^{2} \gamma}{\partial x^{2}}\right) .
\end{array}\right.
$$

Since $v=u+\lambda w$, we now decompose $\dot{v}$ as $\dot{v}=\dot{u}+\lambda \dot{w}+\dot{\lambda} w$. Substituting this decomposition in the second equation of (E1), we have

$$
\frac{\partial \lambda}{\partial t}=\frac{\int_{0}^{L}\left\{-\frac{\partial}{\partial t}\left(\boldsymbol{n} \cdot \gamma^{\prime \prime}\right)\left(u+\lambda w-3\left(\boldsymbol{n} \cdot \gamma^{\prime \prime}\right)^{2}\right)-\left(\boldsymbol{n} \cdot \gamma^{\prime \prime}\right)\left(\frac{\partial u}{\partial t}+\lambda \frac{\partial w}{\partial t}\right)\right\} d x}{L+\int_{0}^{L}\left(\boldsymbol{n} \cdot \gamma^{\prime \prime}\right) w d x}
$$

By these expressions, we shall prove the following lemma. 
Lemma 6.3. For any integer $n \geq 0$, there exist positive constants $C$ and $N$ independent of $t$ such that

$$
\begin{aligned}
\max \left\{\left|\frac{\partial \lambda}{\partial t}\right|, \sup _{x \in S_{L}^{1}}\left|\frac{\partial v}{\partial t}\right|, \sup _{x \in S_{L}^{1}}\left|\frac{\partial}{\partial x} \frac{\partial v}{\partial t}\right|\right\} & \leq C\left(1+\left\|\frac{\partial \gamma}{\partial t}\right\|_{L^{2}}\right)\left\|\frac{\partial \gamma}{\partial t}\right\|_{H^{3}}, \\
\left\|\frac{\partial v}{\partial t}\right\|_{H^{n+2}} & \leq C\left(1+\left\|\frac{\partial \gamma}{\partial t}\right\|_{H^{n}}^{N}\right)\left\|\frac{\partial \gamma}{\partial t}\right\|_{H^{n+3}} .
\end{aligned}
$$

Proof. First, we prove (6.6). By (6.5) and Lemma 5.4, we get $|\dot{\lambda}| \leq C\left(I_{1}+I_{2}\right)$, where

$$
\begin{aligned}
& I_{1}:=\left(\|\dot{\boldsymbol{n}}\|_{L^{2}}\left\|\gamma^{\prime \prime}\right\|_{L^{2}}+\|\boldsymbol{n}\|_{L^{2}}\left\|\dot{\gamma}^{\prime \prime}\right\|_{L^{2}}\right)\left(\sup _{x \in S_{L}^{1}}|u|+|\lambda| \sup _{x \in S_{L}^{1}}|w|+3 \sup _{x \in S_{L}^{1}}\left|\gamma^{\prime \prime}\right|^{2}\right), \\
& I_{2}:=\left\|\gamma^{\prime \prime}\right\|_{L^{2}}\left(\sup _{x \in S_{L}^{1}}|\dot{u}|+|\lambda| \sup _{x \in S_{L}^{1}}|\dot{w}|\right) .
\end{aligned}
$$

By (E2) and Lemma 2.1, we can estimate $\dot{w}, \dot{w}^{\prime}, \dot{u}$, and $\dot{u}^{\prime}$ as follows:

$$
\begin{aligned}
& \max \left\{\sup _{x \in S_{L}^{1}}|\dot{w}|, \sup _{x \in S_{L}^{1}}\left|\dot{w}^{\prime}\right|\right\} \leq C\|\dot{\gamma}\|_{H^{2}} ; \\
& \max \left\{\sup _{x \in S_{L}^{1}}|\dot{u}|, \sup _{x \in S_{L}^{1}}\left|\dot{u}^{\prime}\right|\right\} \leq C\left(1+\|\dot{\gamma}\|_{L^{2}}\right)\|\dot{\gamma}\|_{H^{3}} .
\end{aligned}
$$

Hence, by Lemma 6.1, we estimate $I_{1}$ and $I_{2}$ as follows:

$$
\begin{aligned}
& I_{1} \leq C\left(\|\dot{\gamma}\|_{H^{1}}+\|\dot{\gamma}\|_{H^{2}}\right)\left(1+\|\dot{\gamma}\|_{L^{2}}+\|\dot{\gamma}\|_{L^{2}}^{1 / 2}\right) \leq C\left(1+\|\dot{\gamma}\|_{L^{2}}\right)\|\dot{\gamma}\|_{H^{2}} \\
& I_{2} \leq C\left(1+\|\dot{\gamma}\|_{L^{2}}\right)\|\dot{\gamma}\|_{H^{3}} .
\end{aligned}
$$

Therefore we have an estimate for $\dot{\lambda}$. Since $\dot{v}=\dot{u}+\lambda \dot{w}+\dot{\lambda} w$, by the same arguments, we have (6.6). Finally, we prove (6.7). By Lemmas 2.2 and 6.2,

$$
\begin{aligned}
\|\dot{w}\|_{H^{n+2}} & \leq C\left(1+\left\|\left|\gamma^{\prime \prime}\right|^{2}\right\|_{H^{n}}^{N}\right)\left\|-2\left(\gamma^{\prime \prime} \cdot \dot{\gamma}^{\prime \prime}\right) w-\left(\dot{\boldsymbol{n}} \cdot \gamma^{\prime \prime}+\boldsymbol{n} \cdot \dot{\gamma}^{\prime \prime}\right)\right\|_{H^{n}} \\
& \leq C\left(1+\|\gamma\|_{H^{n+3}}^{N_{1}}\right)\left(\|\gamma\|_{C^{n+2}}\|w\|_{C^{n}}\|\dot{\gamma}\|_{H^{n+2}}+\|\gamma\|_{C^{n+2}}\|\dot{\gamma}\|_{H^{n+2}}\right) \\
& \leq C\left(1+\|\gamma\|_{H^{n+3}}^{N_{1}}\right)\left(\|\gamma\|_{H^{n+3}}\left(1+\|\gamma\|_{H^{n+1}}\right)\|\dot{\gamma}\|_{H^{n+2}}+\|\gamma\|_{H^{n+3}}\|\dot{\gamma}\|_{H^{n+2}}\right) \\
& \leq C\left(1+\|\gamma\|_{H^{n+3}}^{N}\right)\|\dot{\gamma}\|_{H^{n+2}} .
\end{aligned}
$$

Similarly, we have

$$
\begin{aligned}
\|w\|_{H^{n+2}} & \leq C\left(1+\|\gamma\|_{H^{n+2}}^{N_{1}}\right) \\
\|\dot{u}\|_{H^{n+2}} & \leq C\left(1+\|\gamma\|_{H^{n+4}}^{N}\right)\|\dot{\gamma}\|_{H^{n+3}} .
\end{aligned}
$$

By virtue of these inequalities, we obtain (6.7).

Next, we shall prove the following key lemma. 
THE MOTION OF ELASTIC CLOSED CURVES UNDER THE AREA-PRESERVING CONDITION 27

Lemma 6.4. For any integer $n \geq 0$, it holds that

$$
\int_{0}^{\infty}\left\|\frac{\partial^{n}}{\partial x^{n}} \frac{\partial \gamma}{\partial t}\right\|_{L^{2}}^{2} d t<\infty
$$

Furthermore,

$$
\left\|\frac{\partial^{n}}{\partial x^{n}} \frac{\partial \gamma}{\partial t}\right\|_{L^{2}} \longrightarrow 0 \quad \text { as } \quad t \longrightarrow \infty
$$

Proof. We prove the assertion by induction. First we have

$$
\int_{0}^{\infty}\left\|\frac{\partial \gamma}{\partial t}\right\|_{L^{2}}^{2} d t=-\frac{1}{2} \int_{0}^{\infty} \frac{d}{d t}\left\|\frac{\partial^{2} \gamma}{\partial x^{2}}\right\|_{L^{2}}^{2} d t=\frac{1}{2}\left[\left\|\frac{\partial^{2} \gamma}{\partial x^{2}}\right\|_{L^{2}}^{2}\right]_{\infty}^{0}<\infty .
$$

Next, we prove that $\|\dot{\gamma}\|_{L^{2}} \rightarrow 0$ as $t \rightarrow \infty$. Since the solution $\gamma(x, t)$ satisfies two constraints, we have

$$
\begin{aligned}
\frac{1}{2} \frac{d}{d t}\|\dot{\gamma}\|_{L^{2}}^{2} & =\left\langle\dot{\gamma}, \frac{\partial}{\partial t}\left\{-\gamma^{(4)}+\left(\left(v-2\left|\gamma^{\prime \prime}\right|^{2}\right) \gamma^{\prime}\right)^{\prime}+\lambda \boldsymbol{n}\right\}\right\rangle_{L^{2}} \\
& =-\left\|\dot{\gamma}^{\prime \prime}\right\|_{L^{2}}^{2}-\left\langle\dot{\gamma}^{\prime},\left(v-2\left|\gamma^{\prime \prime}\right|^{2}\right) \dot{\gamma}^{\prime}\right\rangle_{L^{2}}+\langle\dot{\gamma}, \lambda \dot{\boldsymbol{n}}\rangle_{L^{2}} \\
& \leq-\left\|\dot{\gamma}^{\prime \prime}\right\|_{L^{2}}^{2}+\left.\sup _{x}|v-2| \gamma^{\prime \prime}\right|^{2}\left|\left\|\dot{\gamma}^{\prime}\right\|_{L^{2}}^{2}+\right| \lambda \mid\|\dot{\gamma}\|_{L^{2}}\left\|\dot{\gamma}^{\prime}\right\|_{L^{2}} \\
& \leq-\left\|\dot{\gamma}^{\prime \prime}\right\|_{L^{2}}^{2}+C\left(1+\|\dot{\gamma}\|_{L^{2}}\right)\left(\left\|\dot{\gamma}^{\prime}\right\|_{L^{2}}^{2}+\|\dot{\gamma}\|_{L^{2}}\left\|\dot{\gamma}^{\prime}\right\|_{L^{2}}\right) \\
& \leq-\left\|\dot{\gamma}^{\prime \prime}\right\|_{L^{2}}^{2}+C\left(1+\|\dot{\gamma}\|_{L^{2}}\right)\|\dot{\gamma}\|_{L^{2}}\left\|\dot{\gamma}^{\prime \prime}\right\|_{L^{2}} \\
& \leq-\frac{1}{2}\left\|\dot{\gamma}^{\prime \prime}\right\|_{L^{2}}^{2}+C\left(1+\|\dot{\gamma}\|_{L^{2}}\right)^{2}\|\dot{\gamma}\|_{L^{2}}^{2} .
\end{aligned}
$$

Therefore

$$
\frac{d}{d t}\|\dot{\gamma}\|_{L^{2}}^{2} \leq C\left(1+\|\dot{\gamma}\|_{L^{2}}\right)^{2}\|\dot{\gamma}\|_{L^{2}}^{2}
$$

By (6.8) and (6.10), we deduce that $\|\dot{\gamma}\|_{L^{2}} \rightarrow 0$ as $t \rightarrow \infty$. In particular, $\|\dot{\gamma}\|_{L^{2}}$ is bounded. Hence, by (6.9), we have

$$
\frac{1}{2} \frac{d}{d t}\|\dot{\gamma}\|_{L^{2}}^{2} \leq-\frac{1}{2}\left\|\dot{\gamma}^{\prime \prime}\right\|_{L^{2}}^{2}+C\|\dot{\gamma}\|_{L^{2}}^{2}
$$

Integrating the both sides of (6.11) from 0 to $\infty$ with respect to $t$, we obtain

$$
\frac{1}{2} \int_{0}^{\infty}\left\|\dot{\gamma}^{\prime \prime}\right\|_{L^{2}}^{2} d t \leq-\frac{1}{2} \int_{0}^{\infty} \frac{d}{d t}\|\dot{\gamma}\|_{L^{2}}^{2} d t+C \int_{0}^{\infty}\|\dot{\gamma}\|_{L^{2}}^{2} d t<\infty .
$$

Suppose it holds that

$$
\int_{0}^{\infty}\left\|\frac{\partial^{j}}{\partial x^{j}} \frac{\partial \gamma}{\partial t}\right\|_{L^{2}}^{2} d t<\infty, \quad\left\|\frac{\partial^{j-2}}{\partial x^{j-2}} \frac{\partial \gamma}{\partial t}\right\|_{L^{2}} \longrightarrow 0 \quad \text { as } \quad t \longrightarrow \infty
$$

for $2 \leq j \leq 2 m$, where $m \geq 1$. Then we have

$$
\frac{d}{d t}\left\|\dot{\gamma}^{(2 m)}\right\|_{L^{2}}^{2} \leq-\left\|\dot{\gamma}^{(2 m+2)}\right\|_{L^{2}}^{2}+\left\|\frac{\partial}{\partial t}\left(\left(v-2\left|\gamma^{\prime \prime}\right|^{2}\right) \gamma^{\prime}\right)^{(2 m-1)}+\frac{\partial}{\partial t}(\lambda \boldsymbol{n})^{(2 m-2)}\right\|_{L^{2}}^{2}
$$


We estimate the second term of the right-hand side of (6.12). First,

$$
\begin{aligned}
\| \frac{\partial}{\partial t}( & \left.\left(v-2\left|\gamma^{\prime \prime}\right|^{2}\right) \gamma^{\prime}\right)^{(2 m-1)} \|_{L^{2}}^{2} \\
& \leq 2\left\|\left(\frac{\partial}{\partial t}\left(v-2\left|\gamma^{\prime \prime}\right|^{2}\right) \gamma^{\prime}\right)^{(2 m-1)}\right\|_{L^{2}}^{2}+2\left\|\left(\left(v-2\left|\gamma^{\prime \prime}\right|^{2}\right) \dot{\gamma}^{\prime}\right)^{(2 m-1)}\right\|_{L^{2}}^{2} \\
& \leq C\|\gamma\|_{C^{2 m}}^{2}\left\|\frac{\partial}{\partial t}\left(v-2\left|\gamma^{\prime \prime}\right|^{2}\right)\right\|_{H^{2 m-1}}^{2}+C\left\|v-2\left|\gamma^{\prime \prime}\right|^{2}\right\|_{C^{2 m-1}}^{2}\|\dot{\gamma}\|_{H^{2 m}}^{2} .
\end{aligned}
$$

But, by the assumption and Lemmas 6.2 and 6.3, we have $\|\gamma\|_{C^{2 m}} \leq C$ and

$$
\begin{array}{r}
\left\|v-2\left|\gamma^{\prime \prime}\right|^{2}\right\|_{C^{2 m-1}}^{2} \leq C\|v\|_{H^{2 m}}^{2}+C\|\gamma\|_{H^{2 m+2}}^{4} \leq C\left(1+\|\dot{\gamma}\|_{H^{2 m-2}}^{N}\right)<C, \\
\left\|\frac{\partial}{\partial t}\left(v-2\left|\gamma^{\prime \prime}\right|^{2}\right)\right\|_{H^{2 m-1}}^{2} \leq 2\|\dot{v}\|_{H^{2 m-1}}^{2}+C\|\gamma\|_{H^{2 m+1}}^{2}\|\dot{\gamma}\|_{H^{2 m+1}}^{2} \leq C\|\dot{\gamma}\|_{H^{2 m+1}}^{2} .
\end{array}
$$

By these inequalities, we obtain

$$
\begin{aligned}
\left\|\frac{\partial}{\partial t}\left(\left(v-2\left|\gamma^{\prime \prime}\right|^{2}\right) \gamma^{\prime}\right)^{(2 m-1)}\right\|_{L^{2}}^{2} & \leq C\|\dot{\gamma}\|_{H^{2 m+1}}^{2}+C\|\dot{\gamma}\|_{H^{2 m}}^{2} \\
& \leq C\left\|\dot{\gamma}^{(2 m+1)}\right\|_{L^{2}}^{2}+C\|\dot{\gamma}\|_{H^{2 m}}^{2} \\
& \leq C\left\|\dot{\gamma}^{(2 m)}\right\|_{L^{2}}\left\|\dot{\gamma}^{(2 m+2)}\right\|_{L^{2}}+C\|\dot{\gamma}\|_{H^{2 m}}^{2} \\
& \leq \frac{\varepsilon}{2}\left\|\dot{\gamma}^{(2 m+2)}\right\|_{L^{2}}^{2}+\frac{C}{2 \varepsilon}\left\|\dot{\gamma}^{(2 m)}\right\|_{L^{2}}^{2}+C\|\dot{\gamma}\|_{H^{2 m}}^{2} .
\end{aligned}
$$

Next, we estimate $\left\|(\partial / \partial t)(\lambda \boldsymbol{n})^{(2 m-2)}\right\|_{L^{2}}^{2}$. By the same arguments,

$$
\begin{aligned}
\left\|\frac{\partial}{\partial t}(\lambda \boldsymbol{n})^{(2 m-2)}\right\|_{L^{2}}^{2} & \leq 2|\dot{\lambda}|^{2}\left\|\gamma^{(2 m-1)}\right\|_{L^{2}}^{2}+2|\lambda|^{2}\left\|\dot{\gamma}^{(2 m-1)}\right\|_{L^{2}}^{2} \\
& \leq C_{1}\left\|\dot{\gamma}^{(2 m-1)}\right\|_{L^{2}}^{2}+C_{2} \\
& \leq C_{1}\left\|\dot{\gamma}^{(2 m+2)}\right\|_{L^{2}}^{1 / 4}\left\|\dot{\gamma}^{(2 m-2)}\right\|_{L^{2}}^{3 / 4}+C_{2} \\
& \leq \frac{\varepsilon}{8}\left\|\dot{\gamma}^{(2 m+2)}\right\|_{L^{2}}^{2}+\frac{C_{3}}{\varepsilon}\left\|\dot{\gamma}^{(2 m-2)}\right\|_{L^{2}}^{2 / 5}+C_{2} .
\end{aligned}
$$

Consequently, we deduce that

$$
\frac{d}{d t}\left\|\dot{\gamma}^{(2 m)}\right\|_{L^{2}}^{2} \leq\left(-1+\frac{5}{8} \varepsilon\right)\left\|\dot{\gamma}^{(2 m+2)}\right\|_{L^{2}}^{2}+C_{1}\left\|\dot{\gamma}^{(2 m)}\right\|_{L^{2}}^{2}+C_{2} .
$$

By choosing $\varepsilon=4 / 5$, we obtain

$$
\frac{d}{d t}\left\|\dot{\gamma}^{(2 m)}\right\|_{L^{2}}^{2} \leq-\frac{1}{2}\left\|\dot{\gamma}^{(2 m+2)}\right\|_{L^{2}}^{2}+C_{1}\left\|\dot{\gamma}^{(2 m)}\right\|_{L^{2}}^{2}+C_{2} .
$$

By combining the assumption with (6.13), we see that $\left\|\dot{\gamma}^{(2 m)}\right\|_{L^{2}} \rightarrow 0$ as $t \rightarrow \infty$. In particular, $\left\|\dot{\gamma}^{(2 m)}\right\|_{L^{2}}$ is bounded. Therefore, integrating the both sides of (6.13), we obtain

$$
\int_{0}^{\infty}\left\|\frac{\partial^{2 m+2}}{\partial x^{2 m+2}} \frac{\partial \gamma}{\partial t}\right\|_{L^{2}}^{2} d t<\infty .
$$


THE MOTION OF ELASTIC CLOSED CURVES UNDER THE AREA-PRESERVING CONDITION 29 This completes the proof.

To prove the convergence of $\gamma(x, t)$, we make use of the following proposition.

Proposition 6.1 (L. Simon [14], Theorem 3). Let $\hat{\gamma}(x)$ be a solution of (AE). Suppose that $\hat{\gamma}(x)$ is not a circle. Then there are a constant $\theta \in(0,1 / 2)$ and $C_{x}^{4+4 \alpha}$ neighborhood $U$ of $\hat{\gamma}$ such that

$$
\left\|\frac{\partial \gamma}{\partial t}\right\|_{L^{2}} \geq|E(\gamma)-E(\hat{\gamma})|^{1-\theta}
$$

for any $\gamma \in U$.

Proof. We consider the operator $\mathcal{M}: C^{4+4 \alpha}\left(S_{L}^{1}\right) \rightarrow C^{4 \alpha}\left(S_{L}^{1}\right)$ defined by

$$
\mathcal{M}(\gamma)=-\frac{\partial^{4} \gamma}{\partial x^{4}}+\frac{\partial}{\partial x}\left\{\left(v-2\left|\frac{\partial^{2} \gamma}{\partial x^{2}}\right|^{2}\right) \frac{\partial \gamma}{\partial x}\right\}+\lambda \boldsymbol{n},
$$

where $\alpha \in(0,1 / 4)$ and $(v, \lambda)$ is a unique solution of the system

$$
\left\{\begin{array}{c}
-v^{\prime \prime}+\left|\gamma^{\prime \prime}\right|^{2} v=2\left|\gamma^{\prime \prime}\right|^{4}-\left|\gamma^{(3)}\right|^{2}-\lambda \boldsymbol{n} \cdot \gamma^{\prime \prime}, \\
\int_{0}^{L}\left\{\left(\boldsymbol{n} \cdot \gamma^{\prime \prime}\right) v-\left(\boldsymbol{n} \cdot \gamma^{\prime \prime}\right)^{3}+\lambda\right\} d x=0 .
\end{array}\right.
$$

Note that $\mathcal{M}$ satisfies the equality

$$
\langle\mathcal{M}(\xi), \zeta\rangle_{L^{2}}=\left.\frac{d}{d \varepsilon} E(\xi+\varepsilon \zeta)\right|_{\varepsilon=0}
$$

for $\xi, \zeta \in C^{4}\left(S_{L}^{1}\right)$. Let $L_{\hat{\gamma}}$ denote the linearization of $\mathcal{M}$ at $\hat{\gamma}$, i.e.,

$$
L_{\hat{\gamma}}(\phi)=\left.\frac{d}{d \varepsilon} \mathcal{M}(\hat{\gamma}+\varepsilon \phi)\right|_{\varepsilon=0}
$$

for $\phi \in C^{4}\left(S_{L}^{1}\right)$. It follows from (6.14) and the definition of $L_{\hat{\gamma}}$ that $L_{\hat{\gamma}}$ is self-adjoint in the sense that if $\phi, \psi \in C^{\infty}\left(S_{L}^{1}\right)$, then $\left\langle L_{\hat{\gamma}}(\phi), \psi\right\rangle_{L^{2}}=\left\langle\phi, L_{\hat{\gamma}}(\psi)\right\rangle_{L^{2}}$. The operator $L_{\hat{\gamma}}$ is written as

$$
L_{\hat{\gamma}}(\phi)=-\frac{d^{4} \phi}{d x^{4}}+\frac{d}{d x}\left\{\left(v_{\varepsilon}-4 \frac{d^{2} \hat{\gamma}}{d x^{2}} \cdot \frac{d^{2} \phi}{d x^{2}}\right) \frac{d \hat{\gamma}}{d x}+\left(\hat{v}-2\left|\frac{d^{2} \hat{\gamma}}{d x^{2}}\right|\right) \frac{d \phi}{d x}\right\}+\hat{\lambda} R \frac{d \phi}{d x}+\lambda_{\varepsilon} \hat{\boldsymbol{n}}
$$

where $(\hat{v}, \hat{\lambda})$ is a solution to the system

$$
\left\{\begin{array}{l}
-\hat{v}^{\prime \prime}+\left|\hat{\gamma}^{\prime \prime}\right|^{2} \hat{v}=2\left|\hat{\gamma}^{\prime \prime}\right|^{4}-\left|\hat{\gamma}^{(3)}\right|^{2}-\hat{\lambda} \hat{\boldsymbol{n}} \cdot \hat{\gamma}^{\prime \prime}, \\
\int_{0}^{L}\left\{\left(\hat{\boldsymbol{n}} \cdot \hat{\gamma}^{\prime \prime}\right) \hat{v}-\left(\hat{\boldsymbol{n}} \cdot \hat{\gamma}^{\prime \prime}\right)^{3}+\hat{\lambda}\right\} d x=0,
\end{array}\right.
$$

and $\left(v_{\varepsilon}, \lambda_{\varepsilon}\right)$ is a solution of the system

$$
\left\{\begin{array}{c}
-v_{\varepsilon}^{\prime \prime}+\left|\hat{\gamma}^{\prime \prime}\right|^{2} v_{\varepsilon}=-2\left(\hat{\gamma}^{\prime \prime} \cdot \phi^{\prime \prime}\right)\left(\hat{v}-4\left|\hat{\gamma}^{\prime \prime}\right|^{2}\right)-2 \hat{\gamma}^{(3)} \cdot \phi^{(3)} \\
\quad-\hat{\lambda}\left(R \phi^{\prime} \cdot \hat{\gamma}^{\prime \prime}+\hat{\boldsymbol{n}} \cdot \phi^{\prime \prime}\right)-\lambda_{\varepsilon} \hat{\boldsymbol{n}} \cdot \hat{\gamma}^{\prime \prime}, \\
\begin{array}{r}
\int_{0}^{L}\left\{\left(\hat{\boldsymbol{n}} \cdot \hat{\gamma}^{\prime \prime}\right) v_{\varepsilon}+\left(R \phi^{\prime} \cdot \hat{\gamma}^{\prime \prime}\right) \hat{v}+\left(\hat{\boldsymbol{n}} \cdot \phi^{\prime \prime}\right) \hat{v}\right. \\
\left.-3\left(\hat{\boldsymbol{n}} \cdot \hat{\gamma}^{\prime \prime}\right)\left\{\left(\hat{\boldsymbol{n}} \cdot \phi^{\prime \prime}\right)+\left(R \phi^{\prime} \cdot \hat{\gamma}^{\prime \prime}\right)\right\}+\lambda_{\varepsilon}\right\} d x=0 .
\end{array}
\end{array}\right.
$$


Note that $(\hat{v}, \hat{\lambda})$ and $\left(v_{\varepsilon}, \lambda_{\varepsilon}\right)$ are uniquely determined, for $\hat{\gamma}$ is not a circle. Moreover $\left(v_{\varepsilon}, \lambda_{\varepsilon}\right)$ is linear with respect to $\phi$ and satisfies $\max \left\{\sup _{x}\left|v_{\varepsilon}\right|, \sup _{x}\left|v_{\varepsilon}^{\prime}\right|,\left|\lambda_{\varepsilon}\right|\right\} \leq C\|\phi\|_{H^{3}}$. Then, we can prove this proposition along the same line as in [14].

By using Proposition 6.1 and Lemma 6.4, we can prove the following theorem:

Theorem 6.1. Let $\gamma_{0}(x)$ be a smooth closed curve with length $L$, enclosed area $\mathcal{A}_{0}$ and rotation number 1 . Assume that $\gamma_{0}(x)$ is not a circle. Then the solution $(\gamma(x, t), v(x, t), \lambda(t))$ of $(\mathrm{GT})$ converges to a solution $(\hat{\gamma}(x), \hat{v}(x), \hat{\lambda})$ of $(\mathrm{AE})$ in the $C^{\infty}$ topology as $t \rightarrow \infty$.

Proof. First, by virtue of Lemmas 6.2 and 6.4, we see that $\|\gamma\|_{H^{n+4}} \leq C$. By Lemma 2.3, we have $\sup _{x \in S_{L}^{1}}\left|\gamma^{(n)}\right| \leq C\|\gamma\|_{H^{n+1}}$. Thus, the solution $\gamma(x, t)$ is bounded in the $C^{\infty}$ topology. Furthermore, it holds that $|\gamma(x)-\gamma(y)| \leq \sqrt{|x-y|}\|\gamma\|_{H^{1}}$. Therefore, it is easy to check that there exists $\left\{t_{j}\right\}_{j}$ such that $t_{j} \rightarrow \infty$ and

$$
\begin{aligned}
& \gamma_{j} \longrightarrow \hat{\gamma}, v_{j} \longrightarrow \hat{v} \quad \text { as } j \longrightarrow \infty \text { in the } C^{\infty} \text { topology } \\
& \lambda_{j} \longrightarrow \hat{\lambda} \text { as } j \longrightarrow \infty
\end{aligned}
$$

where $\gamma_{j}(\cdot):=\gamma\left(\cdot, t_{j}\right), v_{j}(\cdot):=v\left(\cdot, t_{j}\right)$ and $\lambda_{j}:=\lambda\left(t_{j}\right)$. Since $(\gamma, v, \lambda)$ satisfies $(\mathrm{GT})$, by Lemma $6.4,(\hat{\gamma}, \hat{v}, \hat{\lambda})$ satisfies (AE).

Next, we have to show that $\gamma$ converges to $\hat{\gamma}$ in the $C^{\infty}$ topology. But, by using Proposition 6.1, we can prove this assertion in the same way as in [5].

We thus have completed the proof of Theorem 1.1. Concerning the case where $\gamma_{0}(x)$ is a circle, we have stated the result in Proposition 1.1. We close the paper by giving the proof of Proposition 1.1.

Proof of Proposition 1.1. First, we show that $\gamma_{0}(x)$ satisfies (AE). Since $\kappa=1 / r$, by Lemma 4.1, we see that $w_{0}=-r, u_{0}=1 / r^{2}$, and hence $v_{0}=\left(1 / r^{2}\right)-\lambda_{0} r$, where $\lambda_{0}$ is any continuous function. Then it is easy to see that

$$
-\gamma_{0}{ }^{(4)}+\left\{\left(v_{0}-2\left|\gamma_{0}{ }^{\prime \prime}\right|^{2}\right) \gamma_{0}^{\prime}\right\}^{\prime}+\lambda_{0} \boldsymbol{n}_{0}=0
$$

Thus $\gamma_{0}$ satisfies (AE). Second, we claim that the circle obtained by translating and rotating $\gamma_{0}$ is not a solution of the initial value problem. Let us set $\tilde{\gamma_{0}}=\vec{C}(t)+\gamma_{0}(x+\omega(t))$, where $\vec{C}(t)$ is a vector valued function corresponding to translation and $\omega(t)$ is a scalar function corresponding to rotation. If $\tilde{\gamma}_{0}$ satisfies $(\mathrm{AE})$, then $\vec{C}(t)$ is a constant vector and $\omega(t)$ is a constant. However, $\tilde{\gamma}_{0}(x)=\vec{C}+\gamma_{0}(x+\omega)$ does not satisfy the initial condition unless $\vec{C}=0$ and $\omega=0$. Therefore we have completed the proof.

\section{REFERENCES}

[1] P. B. Canham, The minimum energy of bending as a possible explanation of the biconcave shape of the red blood cell, J. Theor. Biol. 26 (1970), 61-81.

[2] J. E. Flaherty, J. B. Keller, and S. I. Rubinow, Post buckling behavior of elastic tubes and rings with opposite sides in contact, SIAM J. Appl. Math. 23 (1972), 446-455.

[3] D. Henry, Geometric Theory of Semilinear Parabolic Equations, Springer - Verlag, New York, 1981.

[4] Y. Kohsaka and T. Nagasawa, On the existence of the Helfrich flow and its center manifold near spheres, Differential Integral Equation 19 no.2 (2006), 121-142.

[5] N. Koiso, On the motion of a curve towards elastica, in Actes da la Table Ronde de Géometrie Différentielle (Luminy 1992), Sémin Congr. 1 Soc. Math. France, Paris (1996), 403-436.

[6] J. Langer and D.A. Singer, Knotted elastic curve in $\mathbb{R}^{3}$, J. London Math. Soc. 30 (1984), 512-520. 
[7] _ - The total squared curvature of closed curves, J. Diff. Geom. 20 (1984), 1-22.

[8] _ Curve straigtening and a minimax argument for closed elastic curves, Topology 24 (1985), 75-88.

[9] A. Linnér, Some properties of the curve straightening flow in the plane, Trans. Amer. Math. Soc. 314 (1989), 605-618.

[10] _ Symmetrized curve-straightening, Differential Geom. Appl. 18 (2003), 119-146.

[11] W. Matsumoto, M. Murai, and S. Yotsutani, By which kind of sound, can one hear the shape of drum?, RIMS Kokyuroku 1315 (2003), 156-175.

[12] A. Pazy, Semigroups of Linear Operators and Applications to Partial Differential Equations, Springer - Verlag, New York, 1983.

[13] A. Polden, Curves and surfaces of least total curvature and fourth-order flows, Universität Tübingen (1996).

[14] L. Simon, Asymptotics for a class of non-linear evolution equations, with applications to geometric problems, Ann. Math 118 (1983), 525-571.

[15] I. Tadjbakhsh and F. Odeh, Equilibrium states of elastic rings, J. Math. Anal. Appl. 18 (1967), 59-74.

[16] Y. Wen, $L^{2}$ flow of curve straightening in the plane, Duke Math. J. 70 (1993), 683-698.

[17] _ Curve straightening flow deforms closed plane curves with nonzero rotation number to circles, J. Diff. Equ. 120 (1995), 89-107.

[18] K. Watanabe, Plane domains which are spectrally determined, Annals of Global Analysis and Geometry 18 (2000), 447-475.

[19] _ Plane domains which are spectrally determined II, J. Inequal. Appl. 7 (2002), 25-47.

Department of Mathematics, Hokkaido University, Sapporo 060-0810, Japan

E-mail address: okabe@math.sci.hokudai.ac.jp 